\title{
Viscous symmetric stability of circular flows
}

\author{
R. C. KLOOSTERZIEL† \\ School of Ocean and Earth Science and Technology, University of Hawaii, Honolulu, HI 96822, USA
}

(Received 20 July 2009; revised 18 December 2009; accepted 20 December 2009)

The linear stability properties of viscous circular flows in a rotating environment are studied with respect to symmetric perturbations. Through the use of an effective energy or Lyapunov functional, we derive sufficient conditions for Lyapunov stability with respect to such perturbations. For circular flows with swirl velocity $V(r)$ we find that Lyapunov stability is determined by the properties of the function $\mathscr{F}(r)=$ $(2 V / r+f) / Q$ (with $f$ the Coriolis parameter, $r$ the radius and $Q$ the absolute vorticity) instead of the customary Rayleigh discriminant $\Phi(r)=(2 V / r+f) Q$. The conditions for stability are valid for flows with non-zero $Q$ everywhere. Further, the flows are presumed stationary, incompressible and velocity perturbations are required to vanish at rigid boundaries. For Lyapunov stable flows an upper bound for the increase of the total perturbation energy due to transient non-modal growth is derived which is valid for any Reynolds number. The theory is applied to Couette flow and the Lamb-Oseen vortex.

\section{Introduction}

In Kloosterziel \& Carnevale (2007) we studied the linear stability of some simple baroclinic parallel shear flows in rotating stratified systems through the use of appropriate Lyapunov functionals. The flows were assumed zonally invariant and subjected to perturbations that are also zonally invariant. The instability that may or may not ensue is sometimes called inertial instability. It is closely related to centrifugal instability, which is a well-known robust phenomenon observed in circular flows subjected to perturbations that are also circularly symmetric. In the laboratory centrifugal instability manifests itself, for example, as the famous axisymmetric Taylor vortices in Couette flow, i.e. the flow between two co-axial rotating cylinders. Because of the zonal invariance of disturbances in parallel shear flows and the circularly symmetric nature of the disturbances of circular flows, both instabilities are appropriately called symmetric instability. In the older meteorological literature it is sometimes called dynamic instability. For graphs from numerical simulations showing the toroidal overturning motions associated with the centrifugal instability in vortices and their fully nonlinear evolution (see e.g. Kloosterziel, Carnevale \& Orlandi 2007). A sketch of the overturning motions associated with the toroidal vortices is provided in figure 1(a). Unlike in Kloosterziel \& Carnevale (2007), in this paper we consider the more complicated question of stability of 'arbitrary' circular flows but we restrict ourselves to flows in a homogeneous fluid. 


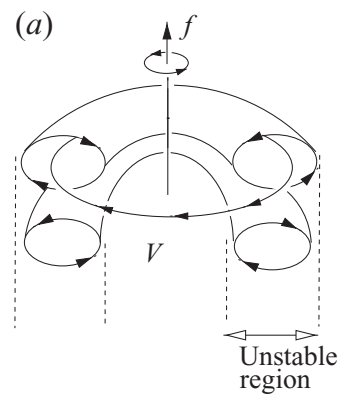

(b)

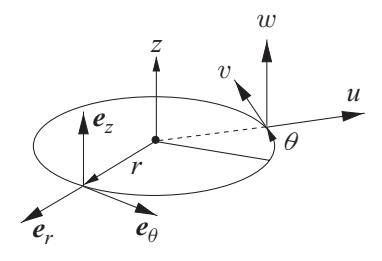

FIGURE 1. (a) Symmetric instability starts in an unstable region as toroidal vortices ('rib vortices') of alternating sign. For a barotropic vortex with swirl velocity $V(r)$ the unstable region is where the Rayleigh discriminant $\Phi(r)<0$ with $\Phi(r)$ defined in (1.1). (b) Diagram defining the polar coordinate system and velocity components for a circular vortex. Here $v$ is the swirl velocity, $w$ the vertical velocity component and $u$ the radial velocity component.

For arbitrary barotropic circular flows with swirl velocity $V(r)$, the inviscid classical condition for stability is that for all $r$ :

$\Phi(r)>0, \quad$ where $\quad \Phi=\left(\frac{2 V}{r}+f\right) Q \quad$ and $\quad Q=\frac{\mathrm{d} V}{\mathrm{~d} r}+\frac{V}{r}+f=\omega+f$

is the absolute vorticity. The inviscid classical condition for instability is that $\Phi(r)<$ 0 in some region. The cylindrical polar coordinate system $(r, \theta, z)$ is sketched in figure $1(b)$. The relative vorticity component $\omega$ is in the $z$ direction and aligned with the axis of rotation and gravitational acceleration. At the time unfamiliar with the older meteorological literature, Kloosterziel \& van Heijst (1991) 'rediscovered' the criterion in an attempt to explain the difficulty in creating stable anticyclones in a rotating fluid in the laboratory. When $f=0$ the condition $\Phi(r)<0$ reduces to Rayleigh's celebrated circulation criterion (Rayleigh 1916) for symmetric instability (see Drazin \& Reid 1981). In an extension of Bayly's analysis (Bayly 1988) to rotating systems, Sipp \& Jacquin (2000) generalized the criterion to non-circular flows. In $\Phi$ the term $2 V / r$ is replaced by $2|V| / \mathscr{R}$, with $|V|$ the velocity amplitude along a streamline and $\mathscr{R}$ the local algebraic radius of curvature of the streamline. If there are streamlines along which $\Phi<0$, then locally instability is guaranteed.

The classical criterion (1.1) for barotropic circular flows in a homogeneous fluid follows from the classical condition for baroclinic circular flows with swirl velocity $V(r, z)$ in a stably stratified fluid. If the fluid is Boussinesq, the criterion for stability is that everywhere in the domain:

$$
N^{2}+\Phi>0 \quad \text { and } \quad N^{2} \Phi-S^{2}>0, \quad \text { where } \quad S=\left(\frac{2 V}{r}+f\right) \frac{\partial V}{\partial z},
$$

and $N^{2}$ the square of the buoyancy frequency. Both criteria (1.1) and the general criterion (1.2) have long been known to meteorologists (see e.g. Sawyer 1947; van Mieghem 1951; Eliassen \& Kleinschmidt 1957; Charney 1973). The second condition in (1.2) appears in various disguises in the literature. The criterion for symmetric stability of barotropic and baroclinic parallel shear flows in a rotating system follow quickly by discarding the curvature term $2 \mathrm{~V} / \mathrm{r}$ in $\Phi$ and for baroclinic flows in $S$ as well. 
The classical stability criterion (1.2) for baroclinic flows pertains only to inviscid and adiabatic fluids and can be established in a variety of well-known ways. For instance, it can be established by including rotation and stratification in Rayleigh's fluid ring exchange argument for circular vortices (Rayleigh 1916). The 'pressureless' Lagrangian displacement argument of Solberg (1936) also leads to the stability condition. Fjørtoft (1950) used an energy method which, unlike in Rayleigh's or Solberg's approach, takes continuity, boundary conditions and pressure perturbations into account. Using the fact that for symmetric perturbations the angular momentum or circulation of individual fluid rings is conserved, Fjørtoft showed that the combination of total kinetic energy associated with the azimuthal (swirl) velocity and the potential energy forms an effective potential energy for motions in the meridional $r z$ plane. This effective potential energy depends only on the meridional displacement field. Fjørtoft further showed that the effective potential energy for a given basic flow is a minimum if the criterion for stability (1.2) is satisfied. If so, it follows that the increase in the total meridional kinetic energy can be kept arbitrarily small if the initial velocity perturbations and initial meridional displacements are sufficiently small.

Through a consideration of the time evolution of certain volume integrated quantities, Ooyama (1966) also found that in the linearized dynamics the total meridional kinetic energy and the meridional displacement field can be kept arbitrarily small if the classical condition for stability (1.2) is satisfied. If not, then initial perturbations can be introduced which lead to unbounded growth of both integral quantities, i.e. there will be instability. For the instability proof, Ooyama used a clever construction having noted that the assumption of the existence of normalmodes solutions may be erroneous, i.e. generally the linearized dynamics allows for a normal-modes analysis only when the boundaries have a special shape, which differs for different flows (see Høiland 1962; Yanai \& Tokiaka 1969). Thus, normalmodes stability/instability or so-called exponential stability/instability can rarely be expected to be established for enclosed flows. If the question of the existence of normal-modes solutions is disregarded, a normal-modes analysis does however lead to the inviscid criterion for stability. For barotropic flows in a homogeneous fluid this is shown in the Appendix, i.e. if (1.1) holds, there will be normal-modes stability.

For baroclinic parallel shear flows in a rotating system, Cho, Shepherd \& Vladimirov (1993) as well as $\mathrm{Mu}$, Shepherd \& Swanson (1996) showed that nonlinear stability in the sense of Lyapunov follows if (1.2) is satisfied. They used the energy-Casimir methodology of Fjørtoft (1950) and Arnol'd (1966). It requires the introduction of a 'pseudo-energy'. The difference between the pseudo-energy of the perturbed flow and the basic unperturbed state is the disturbance pseudo-energy which is conserved in the fully nonlinear dynamics. If (1.2) is satisfied, the disturbance pseudo-energy is a positive-definite functional of the finite-amplitude perturbations which implies Lyapunov stability.

In Kloosterziel \& Carnevale (2007) it was shown how the presumed symmetry in the linear perturbation problem allows for the construction of an 'effective energy' $\mathscr{E}$ which also establishes stability in the sense of Lyapunov if the classical condition (1.2) is satisfied. The disturbance pseudo-energy of Cho et al. (1993) reduces in the smallamplitude limit to this effective energy. The stability proofs of Fjørtoft (1950), Ooyama (1966) and Cho et al. (1993) all depend on conservation of angular momentum or absolute velocity as well as density of individual fluid rings or rods. If viscosity and density diffusion are included, these conservation laws no longer hold. But, the 
construction of Kloosterziel \& Carnevale (2007) allows for the inclusion of viscosity and density diffusion (albeit just for the linearized dynamics). With their approach they rediscovered McIntyre's stability boundary for 'double diffusive' instability (McIntyre 1970). McIntyre found this stability boundary with a normal-modes analysis on an unbounded domain (approximating a circular flow by a rectilinear flow with constant vertical and horizontal shear and embedded in a fluid with constant buoyancy frequency) in the limit of vanishing viscosity. This favourable comparison led us to expect that by using Kloosterziel and Carnevale's approach (Kloosterziel $\&$ Carnevale 2007) we might be able to extract useful information regarding stability of more realistic viscous/diffusive flows. The first step in this direction is taken in this paper by considering arbitrary circular flows in a homogeneous fluid.

The plan of this paper is as follows. Section 2 starts with the linear perturbation equations. In $\S 2.1$ we construct the effective energy $\mathscr{E}$ which is conserved in the inviscid linear dynamics, i.e. $\mathrm{d} \mathscr{E} / \mathrm{d} t=0$, where $t$ is time. The construction is only valid for flows for which the (absolute) vorticity $Q$ is sign-definite, i.e. for flows with $Q \neq 0$ everywhere. The effective energy is positive definite if the function

$$
\mathscr{F}(r)=\frac{2 V / r+f}{\mathrm{~d} V / \mathrm{d} r+V / r+f}=\frac{2 V / r+f}{Q}
$$

is positive everywhere and $\mathscr{E}$ then becomes a Lyapunov functional with which stability is investigated throughout this paper. It follows that any flow that satisfies the inviscid classical criterion for stability ( $\Phi$ or $\mathscr{F}>0$ everywhere) is stable in the sense of Lyapunov in the inviscid problem.

In $\S 2.2$ we derive an upper bound for the gain in perturbation energy that may occur due to transient non-modal growth in the inviscid dynamics for Lyapunov stable flows. In $\S 2.3$ we derive two conditions which guarantee that in the viscous dynamics $\mathrm{d} \mathscr{E} / \mathrm{d} t \leqslant 0$ at all times. The first is that if there is a constant $\alpha$ such that for all $r$,

$$
\mathscr{F}(r)>0 \quad \text { and } \quad \mathscr{G}(r ; \alpha) \equiv \frac{\mathrm{d}^{2} \mathscr{F}}{\mathrm{d} r^{2}}+\frac{\alpha}{r} \frac{\mathrm{d} \mathscr{F}}{\mathrm{d} r}-\frac{1}{2}(3-\alpha)(1+\alpha) \frac{\mathscr{F}}{r^{2}} \leqslant 0,
$$

then there is Lyapunov stability. Stability is also guaranteed if everywhere

$$
\mathscr{F}(r)>0 \quad \text { and } \quad\left(\frac{\mathrm{d} \mathscr{F} / \mathrm{d} r}{\mathscr{F}}\right)^{2} \leqslant \frac{4}{r^{2}} .
$$

Either (1.4) or (1.5) is sufficient: they need not both hold simultaneously. Also it is shown that if either of these conditions is met, the inviscid upper bound on the gain derived in $\$ 2.2$ remains valid for any Reynolds number in the viscous dynamics. The criteria (1.4) and (1.5) are the two notable results in this paper.

In the derivations of these results we ignore 'end-effects', i.e. we ignore the fact that if there is, for example, a rigid bottom and/or top, near these boundaries the $V$ field should vanish if the no-slip condition applies. This inconvenience can be avoided (as is usually done) by imagining the fluid to be of infinite extent in the vertical or by assuming that such end-effects are confined to a thin region near the boundaries. Also, at rigid boundaries the velocity perturbations are required to vanish, i.e. the no-flux and no-slip condition are prescribed. In any case, the boundaries must also be circularly symmetric. Further we assume that the unperturbed flow can be considered stationary. 
In $\S 3$ we apply the theory to Couette flow. We find with (1.4) that Couette flow is Lyapunov stable in the viscous dynamics if the classical inviscid criterion is satisfied. Synge (1938) proved with a normal modes analysis that $\Phi(r)>0$ is sufficient for the viscous problem (normal-modes stability). Wood (1964) later gave a short proof using an effective energy integral like ours. Both proofs depended on that $\Phi \propto 1+$ constant $/ r^{2}$, so that when integrating and differentiating simple powers of $r$ appeared (see also Chandrasekhar 1961, who essentially repeats Synge's analysis). Here we have however general conditions for symmetric stability with which we can test the stability of any flow.

In $\S 4$ we consider the Lamb-Oseen vortex. In $\S 4.1$ we find that in a nonrotating environment $(f=0)$ we cannot prove stability with either (1.4) or (1.5). If placed in a rotating environment $(f \neq 0)$ we discern between the cyclonic and anticyclonic case through the sign of the Rossby number $R o$. For cyclones $(R o>0)$ we find in $\S 4.2$ Lyapunov stability for a finite range of positive Rossby numbers whereas classical stability is guaranteed for all $R o>0$. In $\S 4.3$ we show that for anticyclones both (1.4) and (1.5) imply Lyapunov stability in the entire classically stable range $-1<R o<0$. For the anticyclone we find that for any Reynolds number the perturbation energy can increase at most by a factor of 2 . For the cyclone with large Rossby number $R o=26$ the increase cannot exceed a factor of about 5. The maximum increase is smaller for weaker cyclones, i.e. for smaller Rossby numbers.

In $\S 5$ we conclude with a brief summary and discussion of the main results and mention possible future extensions of this work. In the Appendix we show what the mathematical problems are that arise if a normal-modes analysis is attempted with viscosity included. This could have been sufficient motivation for the approach we have taken in this paper.

\section{Formulation of the linearized problem}

Consider a steady circular flow or vortex $v=V(r)$ that is in cyclo-geostrophic and hydrostatic balance:

$$
\frac{V^{2}}{r}+f V=\frac{1}{\rho} \frac{\partial P}{\partial r}, \quad \frac{1}{\rho} \frac{\partial P}{\partial z}=-g,
$$

where $p=P(r, z)$ is the pressure, $\rho$ is the constant density and $g$ is the gravitational constant with gravity aligned with the axis of rotation (along the $z$-axis as sketched in figure 1) and $f$ the Coriolis parameter representing the effect of rotation in the dynamics. When the kinematic viscosity $v \neq 0$, stationary flows must satisfy

$$
\frac{1}{r} \frac{\mathrm{d}}{\mathrm{d} r} r \frac{\mathrm{d} V}{\mathrm{~d} r}-\frac{V}{r^{2}}=0
$$

(like Couette flow between two concentric cylinders). Nonetheless, when we perform a stability analysis below in $\S 2.3$ with viscous effects included, we will treat the basic state as stationary. Whether this leads to conclusions one can have confidence in, largely depends on whether the time scales of the evolution of the basic flow and those of the perturbations are well separated. One can also envision the possibility of introducing an appropriate circularly symmetric external force field acting in the azimuthal direction which renders the azimuthal $v$ field stationary (see $\S 5$ ). 
Introducing perturbations $u, v, w$ and $p$ independent of the azimuthal angle $\theta$ and linearizing about the balanced state (2.1) we get

$$
\begin{aligned}
\left(\frac{\partial}{\partial t}-v \Delta_{1}\right) u-\left(\frac{2 V}{r}+f\right) v & =-\frac{1}{\rho} \frac{\partial p}{\partial r}, \\
\left(\frac{\partial}{\partial t}-v \Delta_{1}\right) v+\left(\frac{\mathrm{d} V}{\mathrm{~d} r}+\frac{V}{r}+f\right) u & =0, \\
\left(\frac{\partial}{\partial t}-v \Delta\right) w & =-\frac{1}{\rho} \frac{\partial p}{\partial z}, \\
\nabla \cdot \boldsymbol{u}=\frac{1}{r} \frac{\partial(r u)}{\partial r}+\frac{\partial w}{\partial z} & =0,
\end{aligned}
$$

where

$$
\Delta=\frac{1}{r} \frac{\partial}{\partial r} r \frac{\partial}{\partial r}+\frac{\partial^{2}}{\partial z^{2}} \quad \text { and } \quad \Delta_{1}=\Delta-\frac{1}{r^{2}} .
$$

In what follows, it will also be useful to write

$$
\Delta_{1}=\frac{\partial}{\partial r} \frac{1}{r} \frac{\partial}{\partial r} r+\frac{\partial^{2}}{\partial z^{2}}
$$

Adding $u \times(2.2)+w \times(2.4)$ we get

$$
\frac{\partial}{\partial t} \frac{1}{2}\left(u^{2}+w^{2}\right)-\left(\frac{2 V}{r}+f\right) u v=-\boldsymbol{u} \cdot \nabla p / \rho+v\left(u \Delta_{1} u+w \Delta w\right),
$$

where

$$
\nabla=\boldsymbol{e}_{r} \frac{\partial}{\partial r}+\boldsymbol{e}_{z} \frac{\partial}{\partial z}, \quad \boldsymbol{u}=\boldsymbol{e}_{r} u+\boldsymbol{e}_{\theta} v+\boldsymbol{e}_{z} w
$$

With (2.3) it follows that

$$
u=-\left(\partial v / \partial t-v \Delta_{1} v\right) /\left(\frac{\mathrm{d} V}{\mathrm{~d} r}+\frac{V}{r}+f\right)=-\left(\partial v / \partial t-v \Delta_{1} v\right) / Q
$$

provided that the (absolute) vorticity $Q$ defined in (1.1) vanishes nowhere. There will be difficulties, for example, if $f=0$ and $V$ is potential flow (i.e. $V \propto 1 / r$ ), as found outside a spinning cylinder which is placed in a large quiescent basin. In that case our analysis fails because then $Q=0$ so that (2.9) is meaningless. Assuming that $Q \neq 0$ everywhere, we can substitute (2.9) in (2.8) and get

$$
\frac{\partial}{\partial t} \frac{1}{2}\left[u^{2}+w^{2}+\mathscr{F} v^{2}\right]=-\boldsymbol{u} \cdot \nabla p / \rho+v\left(u \Delta_{1} u+w \Delta w+\mathscr{F} v \Delta_{1} v\right)
$$

with $\mathscr{F}(r)$ defined in (1.3).

\subsection{Inviscid dynamics}

If we set $v=0$ and integrate (2.10), we find that

$$
\frac{\mathrm{d} \mathscr{E}}{\mathrm{d} t}=0 \quad \text { with } \quad \mathscr{E}=\frac{1}{2} \int_{\mathscr{V}}\left[u^{2}+w^{2}+\mathscr{F} v^{2}\right] \mathrm{d} \mathscr{V}
$$

provided that $\int_{\mathscr{V}} \operatorname{div}(\boldsymbol{u} p / \rho) \mathrm{d} \mathscr{V}=0 . \mathrm{d} \mathscr{V}=2 \pi r \mathrm{~d} r \mathrm{~d} z$ stands for the volume integral over the domain. This will hold if the flow is either enclosed by rigid boundaries where $\boldsymbol{u} \cdot \boldsymbol{n}=0$ with $\boldsymbol{n}$ the unit vector normal to such boundaries (no flux condition), or can be in a domain unbounded in one or more direction, in which case we require 
that $\boldsymbol{u}$ vanishes 'fast enough' for $r \rightarrow \infty$ if unbounded in the radial direction, or for $z \rightarrow \pm \infty$ if unbounded in the vertical. If there is no interior boundary, things must of course be 'well behaved' at the origin $r=0$. From here on we shall call $\mathscr{E}$ the effective energy.

If $2 V / r+f \neq 0$ everywhere the effective energy can also be written as

$$
\mathscr{E}=\frac{1}{2} \int_{\mathscr{V}}\left[u^{2}+w^{2}+\frac{(2 V / r+f)^{2}}{\Phi} v^{2}\right] \mathrm{d} \mathscr{V} .
$$

We mentioned this result (without a derivation) already in Kloosterziel et al. (2007). Clearly $\mathscr{E}$ is a positive-definite functional if the inviscid classical condition $\Phi>0$ is satisfied everywhere in the domain because then also $\mathscr{F}>0$ everywhere. This establishes Lyapunov stability in the inviscid dynamics, i.e. the perturbation energy $E,\|u\| \equiv\left[\int_{\mathscr{V}} u^{2} \mathrm{~d} \mathscr{V}\right]^{1 / 2},\|w\|$ and $\|v\|$ can be kept arbitrarily small at all times by choosing the initial perturbations 'small enough'. To see this, note that if $\mathscr{F}>0$ everywhere, it follows that if at some initial time (say $t=0$ ) small perturbations are introduced, then at all times (subscripts ' 0 ' indicate $t=0$ )

$$
\begin{gathered}
\mathscr{E}(t)=\mathscr{E}_{0}=\frac{1}{2} \int_{\mathscr{V}}\left[u_{0}^{2}+w_{0}^{2}+\mathscr{F} v_{0}^{2}\right] \mathrm{d} \mathscr{V}>0 \quad \text { and } \\
0 \leqslant \frac{1}{2} \int_{\mathscr{V}}\left(u^{2}+w^{2}\right) \mathrm{d} \mathscr{V} \leqslant \mathscr{E}_{0}, \quad 0 \leqslant \frac{1}{2} \min \{\mathscr{F}\} \int_{\mathscr{V}} v^{2} \mathrm{~d} \mathscr{V} \leqslant \frac{1}{2} \int_{\mathscr{V}} \mathscr{F} v^{2} \mathrm{~d} \mathscr{V} \leqslant \mathscr{E}_{0} .
\end{gathered}
$$

The last inequality implies

$$
0 \leqslant \frac{1}{2} \int_{\mathscr{r}} v^{2} \mathrm{~d} \mathscr{V} \leqslant \frac{\mathscr{E}_{0}}{\min \{\mathscr{F}\}}
$$

From here on ' $\min \{\cdots\}$ ' and ' $\max \{\cdots\}$ ' stand for the minimum and maximum of the function in the domain. The total perturbation energy $E$ is

$$
E=\frac{1}{2} \int_{\mathscr{r}}\left[u^{2}+w^{2}+v^{2}\right] \mathrm{d} \mathscr{V} .
$$

For convenience we have dropped the constant density $\rho$ here. It easily verified that

$$
\left.\begin{array}{rl}
0 \leqslant E \leqslant \frac{\mathscr{E}_{0}}{\min \{\mathscr{F}\}} & \text { if } \quad \min \{\mathscr{F}\} \leqslant 1 \quad \text { and } \\
0 \leqslant E \leqslant \mathscr{E}_{0} & \text { if } \quad \min \{\mathscr{F}\} \geqslant 1 .
\end{array}\right\}
$$

Hence $E$ can be kept arbitrarily small if $\mathscr{F}>0$ everywhere provided that $\mathscr{E}_{0}<\infty$. Finite $\mathscr{E}_{0}$ is guaranteed if $\mathscr{F}<\infty$ everywhere which means that $Q=0$ cannot be allowed.

The perturbation energy evolves according to

$$
\frac{\mathrm{d} E}{\mathrm{~d} t}=-\int_{\mathscr{V}}\left[\left(\frac{\mathrm{d} V}{\mathrm{~d} r}-\frac{V}{r}\right) u v\right] \mathrm{d} \mathscr{V}+v \int_{\mathscr{V}}\left(u \Delta_{1} u+v \Delta_{1} v+w \Delta w\right) \mathrm{d} \mathscr{V} .
$$

If the classical criterion for stability $(\Phi>0$ or $\mathscr{F}>0$ everywhere) is satisfied and $v=0$, clearly one would be hard pressed to establish stability with (2.18). But, (2.17) shows that there will then always be Lyapunov stability.

\subsection{Transient growth}

Although the perturbation energy can be kept arbitrarily small by taking the initial perturbations $u_{0}, v_{0}$ and $w_{0}$ small enough, there can be transient growth $\mathrm{d} E / \mathrm{d} t>0$ 
for some period of time. Much recent research has focused on finding 'optimal perturbations' for a variety of flows that lead to the greatest possible transient amplification of initial perturbations. This transient growth phenomenon can occur in any system where the operators that describe the linearized dynamics are nonnormal, i.e. not self-adjoint so that eigenmodes (as in normal-modes analysis) are not mutually orthogonal. Even in systems that are exponentially stable (normal-modes stable), large growth for some finite time-interval is sometimes possible for large Reynolds numbers (see e.g. Farrell 1988, Butler \& Farrell 1992, Trefethen et al. 1993, Pradeep \& Hussain 2006, Schmidt 2007). An upper bound for the so-called 'gain' $G(t)=E(t) / E_{0}$ can be determined as follows: First note that

$$
\mathscr{E}_{0} \leqslant \frac{1}{2} \int_{\mathscr{V}}\left[u_{0}^{2}+w_{0}^{2}+\max \{\mathscr{F}\} v_{0}^{2}\right] \mathrm{d} \mathscr{V}=\frac{1}{2}\left(\left\|u_{0}\right\|^{2}+\left\|w_{0}\right\|^{2}+\max \{\mathscr{F}\}\left\|v_{0}\right\|^{2}\right) .
$$

Next note that

$$
\left.\begin{array}{rlrl}
\frac{\mathscr{E}_{0}}{E_{0}} \leqslant \frac{\left\|u_{0}\right\|^{2}+\left\|w_{0}\right\|^{2}+\max \{\mathscr{F}\}\left\|v_{0}\right\|^{2}}{\left\|u_{0}\right\|^{2}+\left\|w_{0}\right\|^{2}+\left\|v_{0}\right\|^{2}} & \leqslant \max \{\mathscr{F}\} & \text { when } & \max \{\mathscr{F}\} \geqslant 1, \\
\leqslant 1 & \text { when } & \max \{\mathscr{F}\} \leqslant 1 .
\end{array}\right\}
$$

Dividing both sides of the inequalities in (2.17) by $E_{0}$ yields with (2.20)

$$
\begin{aligned}
E(t) / E_{0}=G(t) \leqslant & G_{\max }=1 / \min \{\mathscr{F}\} & \text { when } \max \{\mathscr{F}\} \leqslant 1, \\
& =\max \{\mathscr{F}\} & \text { when } 1 \leqslant \min \{\mathscr{F}\}, \\
& =\max \{\mathscr{F}\} / \min \{\mathscr{F}\} & \text { when } \min \{\mathscr{F}\} \leqslant 1 \leqslant \max \{\mathscr{F}\} .
\end{aligned}
$$

This upper bound $G_{\max }$ for the gain is valid for any classically stable and therefore Lyapunov stable flow in the inviscid dynamics. If $\mathscr{F} \approx 1$ everywhere, then $G_{\max }$ slightly exceeds unity. If one imagines, for example, that $\mathscr{F}=1$ (take $f=0$ and solid-body rotation $V(r)=\Omega r)$ then $\mathscr{E}=E$ and $G_{\max }=1$ according to $(2.21 c)$. There is neither transient growth nor decay: both $\mathrm{d} E / \mathrm{d} t=0$ and $\mathrm{d} \mathscr{E} / \mathrm{d} t=0$.

One may wonder whether these upper bounds are sharp or not. Consider first the case $0<\mathscr{F} \leqslant 1$ so that according to (2.21a) the upper bound is $G_{\max }=$ $1 / \min \{\mathscr{F}\}$. Imagine that initially there are meridional velocity perturbations $u_{0}, w_{0}$ while $v_{0}=0$. Then $E_{0}=(1 / 2)\left(\left\|u_{0}\right\|^{2}+\left\|w_{0}\right\|^{2}\right)=\mathscr{E}_{0}$. Assume that later the meridional velocity perturbations vanish and instead there is a highly concentrated azimuthal perturbation velocity field $v$ centred about the position $r=r_{\min }$ where $\mathscr{F}\left(r_{\min }\right)=$ $\min \{\mathscr{F}\} \leqslant 1$. Then $E(t)=(1 / 2)\|v(t)\|^{2}$ and by conservation of $\mathscr{E}$ we have $\mathscr{E}(t) \lesssim$ $(1 / 2) \min \{\mathscr{F}\}\|v(t)\|^{2}=\mathscr{E}_{0}=E_{0}$ or $E(t) \lesssim E_{0} / \min \{\mathscr{F}\}$. Then $E(t) / E_{0} \lesssim 1 / \min \{\mathscr{F}\}$ which is $(2.21 a)$. Physically this scenario is plausible if the initial $u, w$ fields are concentrated about $r_{\min }$. In the inviscid dynamics we expect that the upper bound (2.21a), although unattainable, is quite sharp and that maximum amplification is found for initial meridional velocity perturbations located in a narrow region where $\mathscr{F}(r)$ attains it minimum.

The upper bound $(2.21 b)$ for cases with $\mathscr{F} \geqslant 1$ follows likewise by imagining that initially there is an azimuthal perturbation field $v_{0}$ highly concentrated about $r=r_{\max }$ where $\mathscr{F}\left(r_{\max }\right)=\max \{\mathscr{F}\} \geqslant 1$. If $u_{0}=w_{0}=0$ we then have $E_{0}=(1 / 2)\left\|v_{0}\right\|^{2}$ and $\mathscr{E}_{0} \lesssim(1 / 2) \max \{\mathscr{F}\}\left\|v_{0}\right\|^{2}$. If at a later time $v=0$ and there are non-zero meridional velocity perturbations then $E(t)=(1 / 2)\left(\|u(t)\|^{2}+\|w(t)\|^{2}\right)=\mathscr{E}(t)$. But conservation of $\mathscr{E}$ implies $E(t)=\mathscr{E}(t) \lesssim \max \{\mathscr{F}\} E_{0}$ so that $E(t) / E_{0} \lesssim \max \{\mathscr{F}\}$. This suggests 
that the upper bound in $(2.21 b)$ could be approached by choosing an initial $v$ field that is confined to a narrow region about $r=r_{\text {max }}$.

If $\max \{\mathscr{F}\}>1$ and $\min \{\mathscr{F}\}<1$, the upper bound $G_{\max }=\max \{\mathscr{F}\} / \min \{\mathscr{F}\}$ in $(2.21 c)$ is found by imagining that initially there is a $v$ field concentrated in the region about $r_{\max }$ while $u_{0}=w_{0}=0$ and that at a later time again $u=w=0$ while the $v$ field is then concentrated around $r=r_{\text {min }}$. This is not a plausible scenario: it is unlikely that an initial $v$ field concentrated in one region evolves according to (2.2)-(2.4) towards a $v$ field that is concentrated in a different region. Hence for flows with $\min \{\mathscr{F}\} \ll 1 \ll \max \{\mathscr{F}\}, G_{\max }$ in $(2.21 c)$ is probably rather conservative.

\subsection{Viscous dynamics}

We will now derive criteria for viscous flows that guarantee that $\mathscr{E}$ is positive definite while at all times $\mathrm{d} \mathscr{E} / \mathrm{d} t \leqslant 0$. With the assumption that we can treat $V$ as stationary, we have

$$
\frac{\mathrm{d} \mathscr{E}}{\mathrm{d} t}=v \int_{\mathscr{V}}\left(u \Delta_{1} u+w \Delta w\right) \mathrm{d} \mathscr{V}+v \int_{\mathscr{V}} \mathscr{F} v \Delta_{1} v \mathrm{~d} \mathscr{V} .
$$

Again, this is only valid if $\int_{\mathscr{V}} \operatorname{div}(\boldsymbol{u} p / \rho) \mathrm{d} \mathscr{V}=0$. At boundaries we require the perturbations $u, v, w$ to vanish and in any infinite direction they are again required to vanish rapidly enough. Then $(2.22)$ is valid and through partial integration it follows that the first term on the right-hand side in (2.22) becomes

$$
v \int_{\mathscr{V}}\left(u \Delta_{1} u+w \Delta w\right) \mathrm{d} \mathscr{V}=-v \int_{\mathscr{V}}\left(\left|r^{-1} \nabla r u\right|^{2}+|\nabla w|^{2}\right) \mathrm{d} \mathscr{V},
$$

where $|a \nabla b|^{2}=a^{2}\left(\partial_{r} b\right)^{2}+a^{2}\left(\partial_{z} b\right)^{2}$. The term (2.23) would also arise as a part of the usual viscous dissipation of the perturbation energy $E$. Let us evaluate the second term on the right-hand side of (2.22). The part involving the $\partial_{z}^{2}$ operator in $\Delta_{1}$ simply gives the term $v \int_{\mathscr{V}} \mathscr{F} v \partial_{z}^{2} v \mathrm{~d} \mathscr{V}=-v \int_{\mathscr{V}} \mathscr{F}\left(\partial_{z} v\right)^{2} \mathrm{~d} \mathscr{V}$. The part involving the $r$ derivatives can be evaluated in two ways, (A) uses the form $\Delta-1 / r^{2}$ with $\Delta$ as in (2.6) while (B) uses the $r$ operator as in $\Delta_{1}$ in (2.7). Assuming $\mathscr{F}$ to be continuous and twice differentiable, we find through partial integration:

$$
\begin{aligned}
& \text { (A) } \int_{\mathscr{V}} \mathscr{F} v\left(\frac{1}{r} \frac{\partial}{\partial r} r \frac{\partial v}{\partial r}-\frac{v}{r^{2}}\right) \mathrm{d} \mathscr{V}=-\int_{\mathscr{V}} \mathscr{F}\left[\left(\frac{\partial v}{\partial r}\right)^{2}+\left(\frac{v}{r}\right)^{2}\right] \mathrm{d} \mathscr{V} \\
& +\frac{1}{2} \int_{\mathscr{V}} \frac{1}{r^{3}} \frac{\mathrm{d}}{\mathrm{d} r} r \frac{\mathrm{d} \mathscr{F}}{\mathrm{d} r}(r v)^{2} \mathrm{~d} \mathscr{V}, \\
& \text { (B) } \int_{\mathscr{V}} \mathscr{F} v \frac{\partial}{\partial r} \frac{1}{r} \frac{\partial r v}{\partial r} \mathrm{~d} \mathscr{V}=-\int_{\mathscr{V}} \mathscr{F}\left(\frac{1}{r} \frac{\partial r v}{\partial r}\right)^{2} \mathrm{~d} \mathscr{V}+\frac{1}{2} \int_{\mathscr{V}} \frac{1}{r} \frac{\mathrm{d}}{\mathrm{d} r} \frac{1}{r} \frac{\mathrm{d} \mathscr{F}}{\mathrm{d} r}(r v)^{2} \mathrm{~d} \mathscr{V} \text {. }
\end{aligned}
$$

Thus we get

$$
\begin{aligned}
& \text { (A) } \frac{\mathrm{d} \mathscr{E}}{\mathrm{d} t}=-v \int_{\mathscr{V}}\left(\left|r^{-1} \nabla r u\right|^{2}+|\nabla w|^{2}\right) \mathrm{d} \mathscr{V}-v \int_{\mathscr{V}} \mathscr{F}|\nabla v|^{2} \mathrm{~d} \mathscr{V} \\
& +\frac{v}{2} \int_{\mathscr{V}}\left(\frac{\mathrm{d}^{2} \mathscr{F}}{\mathrm{d} r^{2}}+\frac{1}{r} \frac{\mathrm{d} \mathscr{F}}{\mathrm{d} r}-\frac{2 \mathscr{F}}{r^{2}}\right) v^{2} \mathrm{~d} \mathscr{V}, \\
& \text { (B) } \frac{\mathrm{d} \mathscr{E}}{\mathrm{d} t}=-v \int_{\mathscr{V}}\left(\left|r^{-1} \nabla r u\right|^{2}+|\nabla w|^{2}\right) \mathrm{d} \mathscr{V}-v \int_{\mathscr{V}} \mathscr{F}\left|r^{-1} \nabla r v\right|^{2} \mathrm{~d} \mathscr{V} \\
& +\frac{v}{2} \int_{\mathscr{V}}\left(\frac{\mathrm{d}^{2} \mathscr{F}}{\mathrm{d} r^{2}}-\frac{1}{r} \frac{\mathrm{d} \mathscr{F}}{\mathrm{d} r}\right) v^{2} \mathrm{~d} \mathscr{V} \text {. }
\end{aligned}
$$


From this it follows that a sufficient condition for stability is that

$$
\mathscr{F}(r)>0 \quad \text { and } \quad(\mathrm{A}) \quad \frac{\mathrm{d}^{2} \mathscr{F}}{\mathrm{d} r^{2}}+\frac{1}{r} \frac{\mathrm{d} \mathscr{F}}{\mathrm{d} r}-\frac{2 \mathscr{F}}{r^{2}} \leqslant 0 \quad \text { or } \quad \text { (B) } \quad \frac{\mathrm{d}^{2} \mathscr{F}}{\mathrm{d} r^{2}}-\frac{1}{r} \frac{\mathrm{d} \mathscr{F}}{\mathrm{d} r} \leqslant 0,
$$

because then $\mathscr{E}=(1 / 2) \int_{\mathscr{V}}\left(u^{2}+w^{2}+\mathscr{F} v^{2} \mathrm{~d} \mathscr{V}\right)$ is positive definite and at all times the right-hand side in (2.24) or (2.25) is negative or zero and thus $\mathrm{d} \mathscr{E} / \mathrm{d} t \leqslant 0$.

But, this can be improved as follows: Consider (2.24) $-c \times(2.25)$, with $c \neq 1$ a constant:

$$
\begin{aligned}
(1-c) \frac{\mathrm{d} \mathscr{E}}{\mathrm{d} t}= & -(1-c) v \int_{\mathscr{V}}\left\{\left|r^{-1} \nabla r u\right|^{2}+|\nabla w|^{2}+\mathscr{F}\left(\frac{\partial v}{\partial z}\right)^{2}\right\} \mathrm{d} \mathscr{V} \\
& +\frac{v}{2} \int_{\mathscr{r}}\left\{(1-c) \frac{\mathrm{d}^{2} \mathscr{F}}{\mathrm{d} r^{2}}+(1+c) \frac{1}{r} \frac{\mathrm{d} \mathscr{F}}{\mathrm{d} r}\right\} v^{2} \mathrm{~d} \mathscr{V} \\
& -v \int_{\mathscr{V}} \mathscr{F}\left\{(1-c)\left[\left(\frac{\partial v}{\partial r}\right)^{2}+\left(\frac{v}{r}\right)^{2}\right]-2 c \frac{\partial v}{\partial r} \frac{v}{r}\right\} \mathrm{d} \mathscr{V} .
\end{aligned}
$$

The term $\{\cdots\}$ in the last integral on the right-hand side in (2.27) equals

$$
(1-c)\left\{\left(\frac{\partial v}{\partial r}-\left(\frac{c}{1-c}\right) \frac{v}{r}\right)^{2}+\left(1-\frac{c^{2}}{(1-c)^{2}}\right)\left(\frac{v}{r}\right)^{2}\right\} .
$$

We divide both sides of (2.27) by $(1-c)$ and set

$$
\alpha=\frac{1+c}{1-c} \quad \text { so that } \quad 2\left(1-\frac{c^{2}}{(1-c)^{2}}\right)=\frac{1}{2}(3-\alpha)(1+\alpha) \quad \text { and } \quad \frac{c}{c-1}=\frac{1}{2}(\alpha-1) .
$$

The effective energy equation then becomes

$$
\begin{aligned}
\frac{\mathrm{d} \mathscr{E}}{\mathrm{d} t}= & -v \int_{\mathscr{r}}\left\{\left|r^{-1} \nabla r u\right|^{2}+|\nabla w|^{2}+\mathscr{F}\left(\frac{\partial v}{\partial z}\right)^{2}\right\} \mathrm{d} \mathscr{V}-v \int_{\mathscr{V}} \mathscr{F}\left(\frac{\partial v}{\partial r}-\frac{1}{2}(\alpha-1) \frac{v}{r}\right)^{2} \mathrm{~d} \mathscr{V} \\
& +\frac{v}{2} \int_{\mathscr{r}}\left\{\frac{\mathrm{d}^{2} \mathscr{F}}{\mathrm{d} r^{2}}+\frac{\alpha}{r} \frac{\mathrm{d} \mathscr{F}}{\mathrm{d} r}-\frac{1}{2}(3-\alpha)(1+\alpha) \frac{\mathscr{F}}{r^{2}}\right\} v^{2} \mathrm{~d} \mathscr{V} .
\end{aligned}
$$

Therefore a sufficient condition for stability of a viscous flow with respect to arbitrary symmetric perturbations is that, for some constant $\alpha,(1.4)$ is satisfied everywhere. In (1.4) only the range $-1 \leqslant \alpha \leqslant 3$ is useful since only in that range $(3-\alpha)(1+\alpha) \geqslant 0$. For $\alpha=1$ this is condition (A) in (2.26), while (B) is found for $\alpha=-1$.

Stability also follows if $\mathscr{F}>0$ and if

$$
\frac{1}{2} \int_{\mathscr{V}}\left\{\frac{\mathrm{d}^{2} \mathscr{F}}{\mathrm{d} r^{2}}+\frac{\alpha}{r} \frac{\mathrm{d} \mathscr{F}}{\mathrm{d} r}-\frac{1}{2}(3-\alpha)(1+\alpha) \frac{\mathscr{F}}{r^{2}}\right\} v^{2} \mathrm{~d} \mathscr{V}-\int_{\mathscr{V}} \mathscr{F}\left(\frac{\partial v}{\partial r}-\frac{1}{2}(\alpha-1) \frac{v}{r}\right)^{2} \mathrm{~d} \mathscr{V} \leqslant 0 .
$$

If $F>0$ we can substitute $v=\tilde{v} / \sqrt{\mathscr{F}}$ in (2.24), (2.25) or (2.28). Then

$$
\frac{\partial v}{\partial r}=\frac{1}{\mathscr{F}^{1 / 2}} \frac{\partial \tilde{v}}{\partial r}-\frac{1}{2}\left(\frac{\mathrm{d} \mathscr{F} / \mathrm{d} r}{\mathscr{F}^{3 / 2}}\right) \tilde{v}
$$

and if this is substituted in the integral $\int_{\mathscr{V}} \mathscr{F}|\nabla v|^{2} \mathrm{~d} \mathscr{V}$ that appears in (2.24) one gets

$$
\int_{\mathscr{V}} \mathscr{F}|\nabla v|^{2} \mathrm{~d} \mathscr{V}=\int_{\mathscr{V}}\left(\frac{\partial \tilde{v}}{\partial z}\right)^{2} \mathrm{~d} \mathscr{V}+\int_{\mathscr{V}}\left\{\left(\frac{\partial \tilde{v}}{\partial r}\right)^{2}-\frac{\tilde{v}}{\mathscr{F}} \frac{\mathrm{d} \mathscr{F}}{\mathrm{d} r} \frac{\partial \tilde{v}}{\partial r}+\frac{1}{4}\left(\frac{\mathrm{d} \mathscr{F} / \mathrm{d} r}{\mathscr{F}}\right)^{2} \tilde{v}^{2}\right\} \mathrm{d} \mathscr{V} .
$$


Next we use that

$$
-\int_{\mathscr{V}} \frac{\tilde{v}}{\mathscr{F}} \frac{\mathrm{d} \mathscr{F}}{\mathrm{d} r} \frac{\partial \tilde{v}}{\partial r} \mathrm{~d} \mathscr{V}=-\frac{1}{2} \int_{\mathscr{V}} \frac{\partial}{\partial r}\left(\frac{r \tilde{v}^{2}}{\tilde{\mathscr{F}}} \frac{\mathrm{d} \mathscr{F}}{\mathrm{d} r}\right) \mathrm{d} r \mathrm{~d} z+\frac{1}{2} \int_{\mathscr{V}} \frac{\tilde{v}^{2}}{r} \frac{\mathrm{d}}{\mathrm{d} r}\left(\frac{r}{\mathscr{F}} \frac{\mathrm{d} \mathscr{F}}{\mathrm{d} r}\right) \mathrm{d} \mathscr{V} .
$$

Assuming $v$ and therefore $\tilde{v}$ to vanish at boundaries or rapidly enough for large $r$ or at $r=0$, the integral $\int_{\mathscr{V}} \partial / \partial r(\cdots) \mathrm{d} r \mathrm{~d} z=0$ and we find that (2.24) becomes

$$
\begin{aligned}
\frac{\mathrm{d} \mathscr{E}}{\mathrm{d} t}= & -v \int_{\mathscr{V}}\left\{\left|r^{-1} \nabla r u\right|^{2}+|\nabla w|^{2}+\left(\frac{\partial \tilde{v}}{\partial z}\right)^{2}\right\} \mathrm{d} \mathscr{V} \\
& -v \int_{\mathscr{V}}\left\{\left(\frac{\partial \tilde{v}}{\partial r}\right)^{2}-\frac{1}{4}\left[\left(\frac{\mathrm{d} \mathscr{F} / \mathrm{d} r}{\mathscr{F}}\right)^{2}-\frac{4}{r^{2}}\right] \tilde{v}^{2}\right\} \mathrm{d} \mathscr{V} .
\end{aligned}
$$

Equations (2.25) and (2.28) also take this form after the substitution $v=\tilde{v} / \sqrt{\mathscr{F}}$. Hence stability is also guaranteed if (1.5) holds everywhere. One might expect that (1.5) is a less conservative condition than (1.4) since (1.5) follows from combining the second negative-definite integral on the right-hand side of (2.28) with the last integral containing $\mathscr{G}(r ; \alpha)$ which we defined in (1.4). But, as we will see in the next section, this is not necessarily true. In any case, if either (1.4) or (1.5) is satisfied, $\mathrm{d} \mathscr{E} / \mathrm{d} t=0$ only when $\nabla r u, \nabla w, \nabla v$ and $v=0$ everywhere in the domain. If the domain is either confined in the vertical or in the horizontal by rigid boundaries where the perturbations vanish, this implies that $u=v=w=0$ so that $\lim _{t \rightarrow \infty} \mathscr{E}=0$ and $\lim _{t \rightarrow \infty} E=0$. Hence the flow would then be forced back towards the basic flow $V(r)$ and the flow is asymptotically stable.

If either (1.4) or (1.5), or both, are satisfied everywhere, the upper bounds $G_{\max }$ on the gain due to possible transient growth remain $(2.21 a)-(2.21 c)$ because then $\mathrm{d} \mathscr{E} / \mathrm{d} t \leqslant 0$ at all times so that instead of (2.17) we have

$$
\left.\begin{array}{lll}
0 \leqslant E(t) \leqslant \frac{\mathscr{E}(t)}{\min \{\mathscr{F}\}} \leqslant \frac{\mathscr{E}_{0}}{\min \{\mathscr{F}\}} \quad \text { if } & \min \{\mathscr{F}\} \leqslant 1, \\
0 \leqslant E(t) \leqslant \mathscr{E}(t) \leqslant \mathscr{E}_{0} & \text { if } \quad \min \{\mathscr{F}\} \geqslant 1 .
\end{array}\right\}
$$

Division by $E_{0}$ and using (2.20) then again yields $(2.21 a)-(2.21 c)$. For small Reynolds numbers $R e$, these upper bounds are expected to be conservative because the evolution equations (2.28) and (2.30) indicate that the effective energy $\mathscr{E}$ will diminish rapidly for small $R e$ if either (1.4) and/or (1.5) is satisfied everywhere.

\section{Couette flow}

The well-known Couette flow of a viscous fluid between rotating coaxial cylinders has the velocity distribution

$$
V(r)=A r+B / r \quad \text { with } \quad A=\Omega_{1} \frac{\mu-\eta^{2}}{1-\eta^{2}}, \quad B=\Omega_{1} R_{1}^{2} \frac{1-\mu}{1-\eta^{2}}
$$

and the parameters

$$
\mu=\Omega_{2} / \Omega_{1} \quad \text { and } \quad \eta=R_{1} / R_{2} .
$$

$R_{1}$ and $R_{2}$ are the radius of the inner and the outer cylinder which rotate with angular velocity $\Omega_{1}$ and $\Omega_{2}$, respectively (see Drazin \& Reid 1981). The Rayleigh discriminant $\Phi(r)$ (with $f=0$ ) is positive throughout the domain $R_{1} \leqslant r \leqslant R_{2}$ if 
$\mu>\eta^{2}$. This means that for classical (inviscid) stability the cylinders must rotate in the same direction and

$$
\left|V\left(R_{2}\right) R_{2}\right|>\left|V\left(R_{1}\right) R_{1}\right|
$$

We have

$$
\mathscr{F}(r)=1+\frac{B}{A r^{2}}=1+\left(\frac{1-\mu}{\mu-\eta^{2}}\right) \frac{1}{\left(r / R_{1}\right)^{2}}
$$

and $\mathscr{F}(r)>0$ for all $r \in\left[R_{1}, R_{2}\right]$ if the classical criterion $\left(\mu>\eta^{2}\right)$ is satisfied (note that $\Phi(r)=4 A^{2} \mathscr{F}(r)$ for Couette flow). Like every flow that satisfies the classical condition, in the inviscid dynamics Couette flow is Lyapunov stable.

The second condition in (1.4) is after substitution of (3.3)

$$
\mathscr{G}(r ; \alpha)=(6-2 \alpha) \frac{B}{A} \frac{1}{r^{4}}-\frac{1}{2}(3-\alpha)(1+\alpha)\left(\frac{1}{r^{2}}+\frac{B}{A} \frac{1}{r^{4}}\right) \leqslant 0 .
$$

This will be satisfied for $\alpha=3$, i.e. for $\alpha=3$ the last term in (2.28) vanishes identically for all $r>0$. Hence Couette flow is also Lyapunov stable in the viscous dynamics if the inviscid classical criterion is satisfied.

For Couette flow (1.5) is too conservative: we find that the second condition in (1.5) is only satisfied when $\mu<2-\eta^{2}$. This implies that the classical condition plus the second condition in (1.5) require that the inner and outer cylinder rotate in the same direction and that

$$
\left|V\left(R_{2}\right) R_{2}\right|>\left|V\left(R_{1}\right) R_{1}\right| \quad \text { but } \quad\left|V\left(R_{2}\right) R_{2}\right|<\left|V\left(R_{1}\right) R_{1}\right|\left(2\left(R_{2} / R_{1}\right)^{2}-1\right) .
$$

Thus (1.5) only proves Lyapunov stability of a subset of the classically stable Couette flows whereas (1.4) proves stability for all classically stable flows. For different flows the converse may be true, i.e. (1.5) may sometimes be less restrictive than (1.4). This is shown in the next section with an example.

There are two special cases worthwhile mentioning. The first is that of where the inner cylinder is taken out. Then $V=\Omega_{2} r$, which is simply solid-body rotation as found inside a rotating cylinder after a sufficiently long spin-up time. Then $\Phi=4 \Omega_{2}^{2}$ and $\mathscr{F}=1$. This is therefore inviscidly stable to symmetric perturbations because the effective energy is positive-definite, but also Lyapunov stable in the viscous dynamics since either (2.24) or (2.25) show that $\mathrm{d} \mathscr{E} / \mathrm{d} t \leqslant 0$ and also $\mathrm{d} E / \mathrm{d} t \leqslant 0$. The second case is the limit $R_{2} \rightarrow \infty$ and $\Omega_{2}=0(\mu=0$ and $\eta=0)$. The result would be pure potential flow $V=\Omega_{1} R_{1}^{2} / r$ outside a spinning cylinder. This has $\Phi=0$ everywhere because the vorticity $Q$ is zero and $\mathscr{F}$ is undefined. As mentioned in $\S 2$, our approach cannot be used in this case.

\section{Lamb-Oseen vortex}

The so-called Lamb-Oseen vortex or 'Gaussian' vortex has a velocity distribution $V(r)$ and corresponding vorticity $\omega$ given by

$$
V(r)=\frac{\omega_{0} L}{(r / L)}\left[1-\exp \left(-r^{2} / 2 L^{2}\right)\right], \quad \omega(r)=\frac{\mathrm{d} V}{\mathrm{~d} r}+\frac{V}{r}=\omega_{0} \exp \left(-r^{2} / 2 L^{2}\right) .
$$

The radius $r$ has been non-dimensionalized with an arbitrary length scale $L$ and $\omega_{0}$ is the peak vorticity found at $r=0 . V$ and $\omega$ are shown in figure 2(a). Unlike Couette flow, in a freely evolving viscous fluid this is not a steady state solution of the Navier-Stokes equations and $V$ and $\omega$ evolve according to $\partial_{t} V=\nu \Delta_{1} V$ and 
(a)

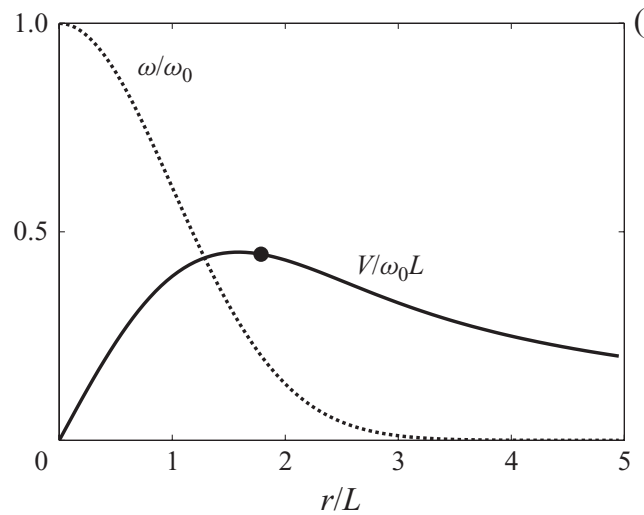

(b)

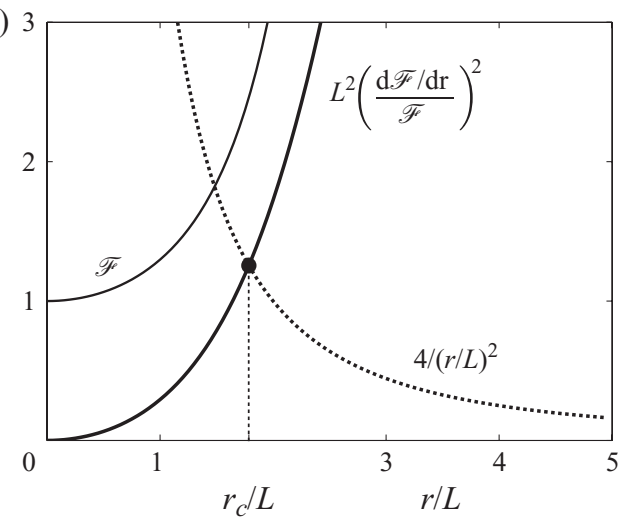

FIGURE 2. (a) The vorticity $\omega$ (stippled line) and velocity $V$ (solid line) of the Lamb-Oseen vortex given by (4.1) non-dimensionalized with $\omega_{0}$ and $\omega_{0} L$, respectively, as a function of $r / L$. Peak vorticity is $\omega_{0}$ at $r=0$ and $L$ is arbitrary. The non-dimensional peak velocity is $V_{\max } /\left(\omega_{0} L\right) \approx 0.45$ at $r_{\max } / L \approx 1.57$. (b) Graph showing $\mathscr{F}$ given by (4.2) (thin line), $(\mathrm{d} \mathscr{F} / \mathrm{d} r)^{2} / \mathscr{F}^{2}$ non-dimensionalized with $L^{2}$ (thick line) and the curve $4 /(r / L)^{2}$ (stippled line) as a function of $r / L$. For $r>r_{c} / L \approx 1.79$ (indicated by $\bullet$ ) the criterion (1.5) is not satisfied. In $(a)$ the symbol $\bullet$ is also shown at the position $r_{c} / L$.

$\partial_{t} \omega=v \Delta \omega$. If (4.1) defined $V$ and $\omega$ at some time $t=0$, the time evolution is

$$
V(r, t)=\frac{\omega_{0}(t) L(t)}{(r / L(t))}\left[1-\exp \left(-r^{2} / 2 L(t)^{2}\right)\right], \quad \omega(r, t)=\omega_{0}(t) \exp \left(-r^{2} / 2 L(t)^{2}\right)
$$

with

$$
\omega_{0}(t)=\frac{\omega_{0}}{1+2\left(v t / L^{2}(0)\right)}, \quad L(t)=\sqrt{L^{2}(0)+2 v t} .
$$

But, we will treat the flow as stationary and only consider $V$ and $\omega$ defined in (4.1).

\subsection{Non-rotating system}

In a non-rotating system $(f=0)$ the Rayleigh discriminant $\Phi=2(V / r) \omega>0$ for all $r$ and

$$
\mathscr{F}(r)=\frac{2 V / r}{\omega}=2 \frac{\exp \left(r^{2} / 2 L^{2}\right)-1}{(r / L)^{2}} \geqslant 1
$$

for all $r \geqslant 0$, as shown in figure $3(b)$. The smallest value $\mathscr{F}=1$ is found in the limit $r \downarrow 0$. Thus the effective energy is positive definite. But Lyapunov stability with respect to symmetric perturbations in the inviscid dynamics cannot be established. The reason is that as $r \rightarrow \infty$ we have $\mathscr{F} \rightarrow \infty$. This is due to the fact that for large $r$ the vorticity $Q=\omega$ vanishes exponentially fast (the vortex gets ever closer to potential flow $V \propto 1 / r$ so that $Q \rightarrow 0$ ). An upper bound $G_{\max }$ for the possible gain due to transient growth in the inviscid dynamics cannot be determined with $(2.21 b)$ unless the flow is terminated flow at some finite $r=r_{c}$. A finite $G_{\max }=\mathscr{F}\left(r_{c}\right)$ can be calculated but it can be made arbitrarily large by increasing $r_{c}$. The fact that $G_{\max }$ is not finite on the infinite domain is perhaps not surprising since it is known that potential flow can support unbounded algebraic growth (see $\S 5$ for a brief discussion).

For the viscous dynamics we need to determine whether (1.4) or (1.5) can hold everywhere. In figure $3(b)$ it is seen that for $r / L>r_{c} / L \approx 1.79$ the criterion (1.5) does not hold, i.e. the second condition in (1.5) is violated. Between $r=0$ and $r=r_{c}$ (1.5) 

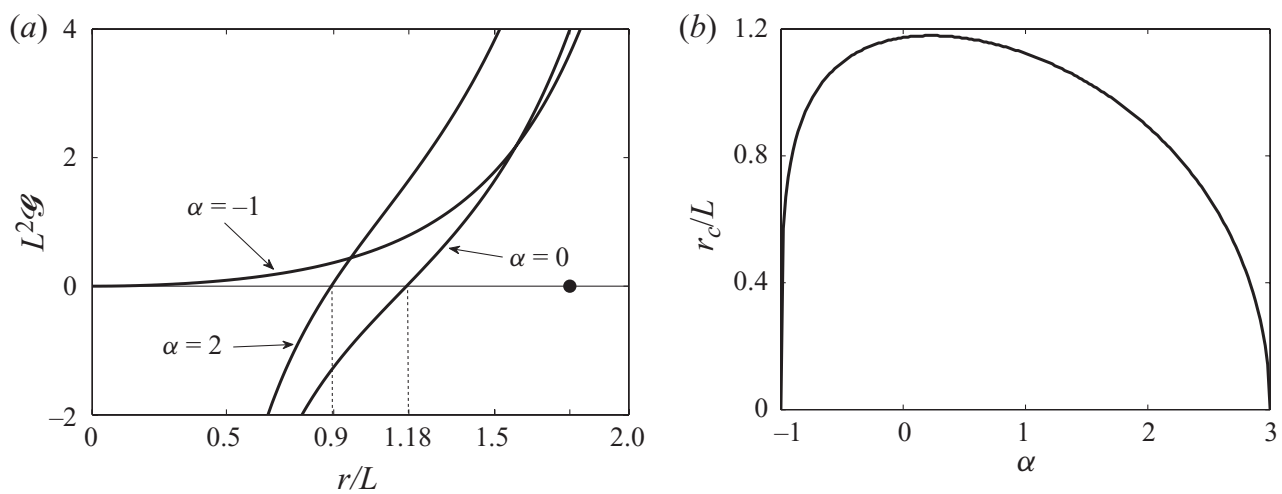

Figure 3. (a) The function $\mathscr{G}(r ; \alpha)$ given in (1.4) and non-dimensionalized with $L^{2}$, for the Lamb-Oseen vortex and $\alpha=-1,0$ and $\alpha=2$. For $\alpha=-1$ and $\alpha=3$ (not shown) $\mathscr{G} \geqslant 0$ for all $r$. For $\alpha=0$ we have $\mathscr{G}>0$ for all $r / L>r_{c} / L \approx 1.18$. (b) Graph showing the critical radius $r_{c} / L$ as a function of $\alpha \in[-1,3]$. The greatest value is found for $\alpha \approx 0.2$ with $r_{c} / L \approx 1.19$. There are no $\alpha$ values for which $\mathscr{G} \leqslant 0$ for all $r$ so (1.4) is never satisfied on the unbounded domain. The symbol $\bullet$ is at the position $r / L=r_{c} / L \approx 1.79$ from figure 3 .

is satisfied. If the flow was terminated at $r=r_{c}$, the flow would be stable but on an unbounded domain Lyapunov stability cannot be established with (1.5).

In figure 3(a) we show $\mathscr{G}(r ; \alpha)$, defined in (1.4), for a few values of $\alpha$. In each case there is a finite value $r=r_{c}$ with $\mathscr{G}>0$ when $r>r_{c}$. In figure $3(b)$ we show the critical value $r_{c} / L$ as a function of $\alpha \in[-1,3]$. There are no $\alpha$ values for which (1.4) is satisfied everywhere. Further, the region where (1.4) can be satisfied is confined to a far smaller range of $r$ values than (1.5): the widest region for which Lyapunov stability can be established according to (1.4) is $r / L \in[0,1.19]$ whereas (1.5) proves stability for the range $r / L \in[0,1.79]$. On a radially unbounded domain we therefore cannot prove Lyapunov stability.

\subsection{Rotating system: cyclones}

We now add the Coriolis force to the dynamics $(f \neq 0)$ and define the Rossby number for the Lamb-Oseen vortex as $R o=\omega_{0} / f$. If $R o>0$ this is a cyclonic vortex, if $R o<0$ it is an anticyclonic vortex. The Rayleigh discriminant non-dimensionalized with $f^{2}$ is

$$
\Phi(r) / f^{2}=\left(2 R o /(r / L)^{2}\left[1-\exp \left(-r^{2} / 2 L^{2}\right)\right]+1\right)\left(R o \exp \left(-r^{2} / 2 L^{2}\right)+1\right),
$$

while

$$
\mathscr{F}=\frac{2 R o /(r / L)^{2}\left[1-\exp \left(-r^{2} / 2 L^{2}\right)\right]+1}{R o \exp \left(-r^{2} / 2 L^{2}\right)+1} .
$$

For all $R o \in(-1, \infty)$ it can be shown that $\Phi(r)>0$ for all $r$ (see Carnevale et al. 1997). For the cyclone $(R o>0)$ this is easily seen with (4.3). Further, for all $R o>-1$ we find that $\mathscr{F}>1 / 2$ for all $r \geqslant 0$. Thus, when $R o>-1$ the effective energy is positive definite and the vortex is therefore Lyapunov stable in the inviscid dynamics for all Rossby numbers $R o>-1$. The smallest value $\mathscr{F}=1 / 2$ is found in the limit $R o \rightarrow-1$ and $r \downarrow 0$. The limiting case $R o \rightarrow-1$ is singular in that the absolute vorticity $Q=\omega+f$ tends to zero in the limit $r \downarrow 0$ so that $\mathscr{F}$ becomes undefined there $($ remember $\mathscr{F}=(2 V / r+f) / Q)$.

For the viscous dynamics, we first consider the cyclone case $R o>0$. In figure $4(a)$ we show $\mathscr{F}$ for a few positive Rossby numbers. Generally for all $R o>0$ we have 

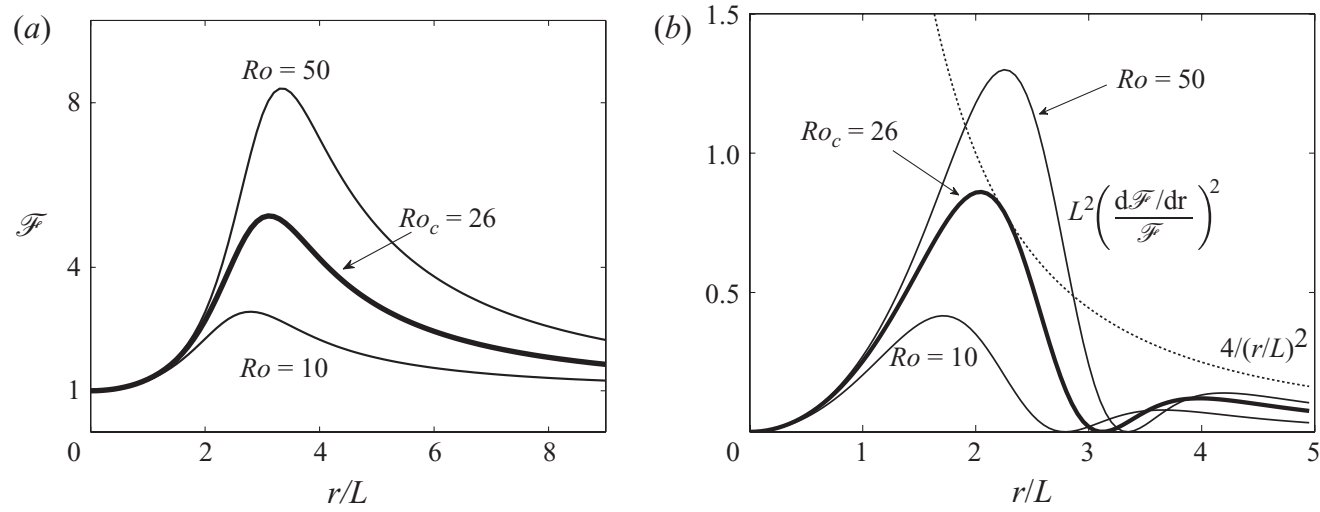

FIGURE 4. (a) $\mathscr{F}$ as a function of $r / L$ for the cyclonic Lamb-Oseen vortex with $R o=\omega_{0} / f=10,26$ (thick line) and $R o=50$. For any $R o>0$ we have $\mathscr{F} \geqslant 1$ for all $r \geqslant 0$. (b) $(\mathrm{d} \mathscr{F} / \mathrm{d} r)^{2} / \mathscr{F}^{2}$ for $R o=10,26$ and 50 and non-dimensionalized with $L^{2}$ (solid lines) and the curve $4 /(r / L)^{2}$ (stippled line) as a function of $r / L$. For $0<R o \leqslant R o_{c} \approx 26$ the criterion (1.5) is satisfied for all $r \geqslant 0$ (numbers are approximate: $26<R o_{c}<26.01$ ). For $R o=R o_{c}$ the curve $L^{2}(\mathrm{~d} \mathscr{F} / \mathrm{d} r)^{2} / \mathscr{F}^{2}$ 'touches' the curve $4 /(r / L)^{2}$ at $r / L \approx 2.25$ (thick line). For $R o>26$ there is a range of $r$ values in which the second condition in (1.5) does not hold. For all $R o<26$ the cyclone is Lyapunov stable according to (1.5).
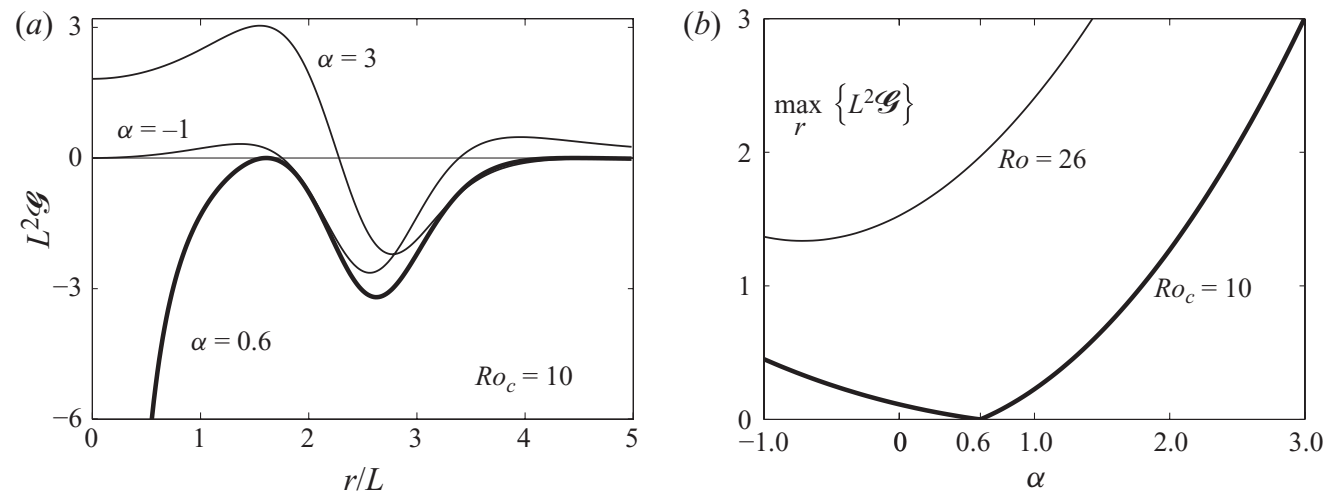

FIGURE 5. (a) The function $\mathscr{G}(r ; \alpha)$ given in (1.4) and non-dimensionalized with $L^{2}$ for the cyclonic Lamb-Oseen vortex with $R o=R o_{c}=10$ and $\alpha=-1,0.6$ (thick line) and $\alpha=3$. $(b)$ Numerically determined non-dimensional maximum of $\mathscr{G}$ for $R o=R o_{c}=10$ and $R o=26$ as a function of $\alpha \in[-1,3]$. For $R o>R o_{c}=10$ the maximum is positive for all $\alpha$ which means that there is always a range of $r$ values where the second condition in (1.4) is violated. For $\alpha \approx 0.6$ the maximum is zero, as shown in $(a)$. For all $R o<R o_{c}$ there is always an $\alpha$ for which $\mathscr{G} \leqslant 0$ for all $r \geqslant 0$. According to (1.4) this guarantees Lyapunov stability.

$\mathscr{F} \geqslant 1$ for all $r$. Numerically we find that for $0<R o \leqslant R o_{c} \approx 26$ the criterion (1.5) is satisfied. This is shown in figure $4(b)$. For Rossby numbers greater that $R o_{c} \approx 26$ the second condition in (1.5) is violated for some range of $r$ values (the precise value lies between 26 and 26.01). Thus, (1.5) establishes Lyapunov stability of the cyclonic Lamb-Oseen vortex only in the range $R o \in(0,26)$ in the viscous dynamics whereas stability is guaranteed for all $R o>0$ in the inviscid dynamics.

The stability criterion (1.4) is satisfied for a smaller range of positive Rossby numbers, i.e. only for $0<R o \lesssim 10$. In figure $5(a)$ we show $\mathscr{G}(r ; \alpha)$ for $R o=R o_{c} \approx 10$ and a few values of $\alpha$ as indicated. For $\alpha \approx 0.6$ we find that $\mathscr{G} \leqslant 0$ for all $r$. This 

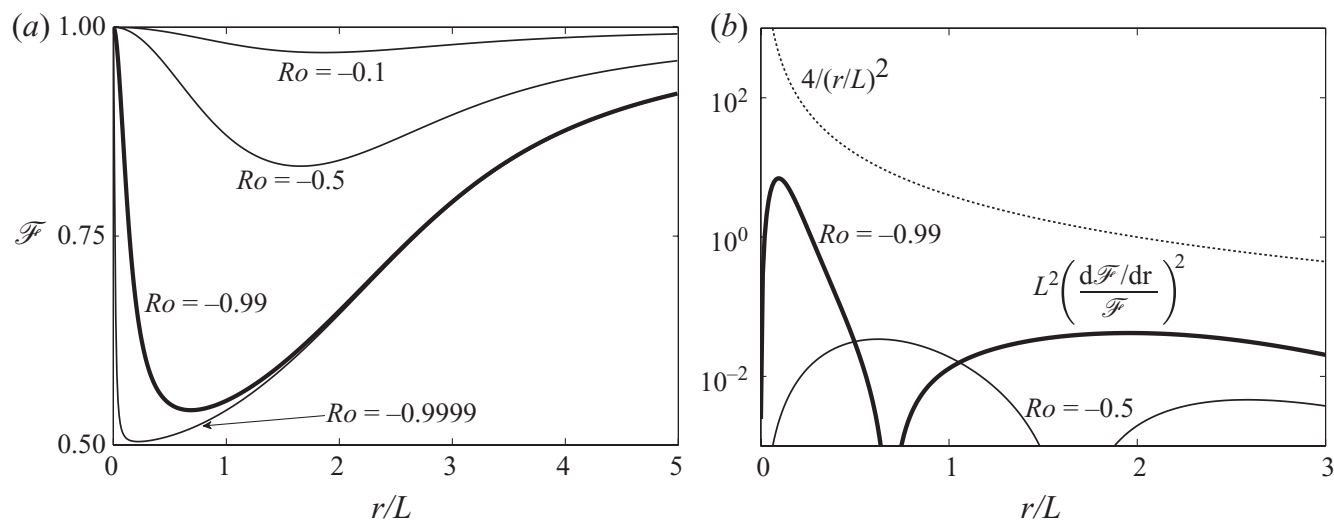

FIGURE 6. (a) $\mathscr{F}$ as a function of $r / L$ for the anticyclonic Lamb-Oseen vortex with $R o=\omega_{0} / f=-0.9999,-0.9,-1 / 2$ and $R o=-1 / 10$. For all $R o>-1$ we find that $\mathscr{F} \geqslant 1 / 2$ for all $r$. $(b)(\mathrm{d} \mathscr{F} / \mathrm{d} r)^{2} / \mathscr{F}^{2}$ non-dimensionalized with $L^{2}$ (solid lines) and the curve $4 /(r / L)^{2}$ (stippled line) as a function of $r / L$. For $-1<R o<0$ the criterion (1.5) is satisfied for all $r \geqslant 0$. This is illustrated here with the examples $R o=-0.9$ (thick line) and $R o=-1 / 2$ (thin line). Also for $R o=-0.9999$ is $L^{2}(\mathrm{~d} \mathscr{F} / \mathrm{d} r)^{2} / \mathscr{F}^{2}<4 /(r / L)^{2}$ for all $r$ (not shown).

is seen in figure $5(a)$ where for $\alpha=0.6 \mathscr{G}$ 'touches' the zero level at some $r$ between $r=1$ and $r=2$, becomes negative again and then asymptotically $\mathscr{G} \rightarrow 0$ for $r \rightarrow \infty$. In this case with $R o \approx 10$, for all $\alpha \neq 0.6$ we find that $\mathscr{G}>0$ for some range of $r$ values. This is illustrated in figure $5(a)$ with two examples. In figure $5(b)$ we show $\max _{r}\{\mathscr{G}\}$ as a function of $\alpha$. For $R o=R o_{c} \approx 10$ we see that $\max _{r}\{\mathscr{G}\}=0$ just for $\alpha \approx 0.6$, while for all other $\alpha$ the maximum is positive. For $R o \gtrsim 10$ we find $\max _{r}\{\mathscr{G}\}>0$ for all $\alpha$ and the second condition in (1.4) can therefore not be satisfied for any $\alpha$ when $R o \gtrsim 10$. This is illustrated in figure $5(b)$ with $R o=26$. For all Rossby numbers $0<R o<R o_{c} \approx 10$, there is always an $\alpha \in[-1,3]$ for which $\mathscr{G} \leqslant 0$ for all $r$ (in each case $\mathscr{G} \rightarrow 0$ as $r \rightarrow \infty$ ). Thus, with (1.5) we find the widest range of positive Rossby numbers $(0<R o \lesssim 26)$ for which we can prove Lyapunov stability in the viscous dynamics.

Contrary to the case of the vortex in a non-rotating environment, we can determine the upper bound on the gain $G_{\max }$. Since for any finite positive Rossby number $1 \leqslant \mathscr{F}<\infty$, according to $(2.21 b)$ we have $G_{\max }=\max \{\mathscr{F}\}$. Figure $8(a)$ shows $G_{\max }$ as a function of the Rossby number in the (viscously) stable range $0<R o \lesssim 26$. For the largest Rossby number $R o=26$ we find that $G_{\max } \approx 5.25$. Since this upper bound is valid for any Reynolds number, including the limit $R e \rightarrow \infty$, this is in marked contrast with the result of Pradeep \& Hussain (2006) who studied the Lamb-Oseen vortex in a non-rotating system. They showed that there can be significant transient growth of axisymmetric perturbations well outside the core of the vortex. A 100-fold increase in the total perturbation energy $E$ (a gain $G=10^{2}$ ) was found even for a modest Reynolds number $R e=2500$, although the flow is normal-modes stable. The difference between the rotating and the non-rotating case is further discussed in $\S 5$.

\subsection{Rotating system: anticyclones}

In the inviscid dynamics the anticyclonic Lamb-Oseen vortex is classically and Lyapunov stable for all Rossby numbers in the range $-1<R o<0$. In figure 6(a) we show $\mathscr{F}$ for a few negative Rossby numbers. For $R o \approx-1$ (illustrated in figure $6 a$ with $R o=-0.9999)$ the smallest possible value $\mathscr{F}=1 / 2$ is approached near $r=0$. The 
(a)

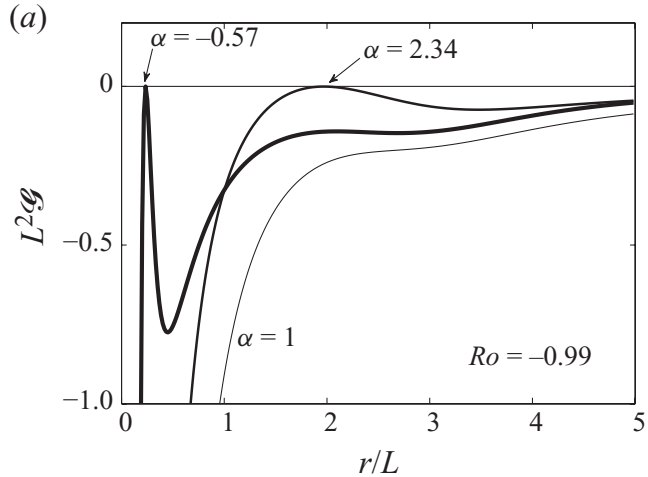

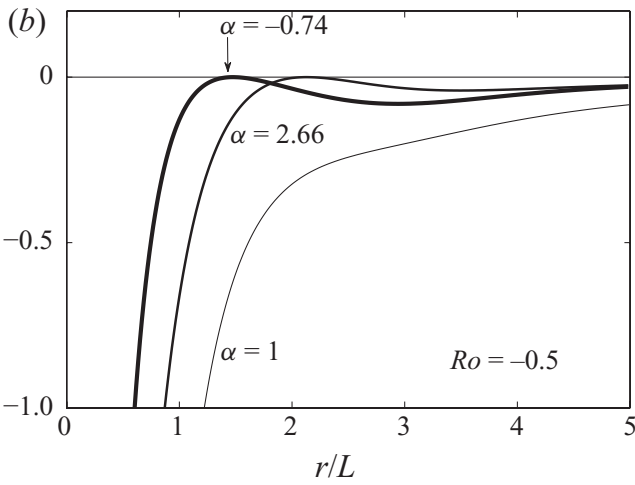

Figure 7. $(a)$ The function $\mathscr{G}$ for the anticyclonic Lamb-Oseen vortex with $R o=-0.99$ and $\alpha$ values as indicated. For all $\alpha \in[-0.57,2.34]$ we find that $\mathscr{G} \leqslant 0$ for all $r$. For the two bounding values of this range $\mathscr{G}$ is shown as a thick line. (b) Same as $(a)$ but for $R o=-1 / 2$ and $\alpha$ values as indicated. For all $\alpha \in[-0.74,2.66]$ we have $\mathscr{G} \leqslant 0$ for all $r$. In both cases the anticyclonic Lamb-Oseen vortex is Lyapunov stable according to (1.4). Generally for any Rossby number $-1<R o<0$ there is a range of $\alpha$ values within the range $[-1,3]$ that yield $\mathscr{G} \leqslant 0$ for all $r$ while $\mathscr{G} \rightarrow 0$ for $r \rightarrow \infty$. The narrowest range is found in the limit $R o \downarrow-1$. For $R o=-0.9999$ it is to three significant digits the same as shown in $(a)$, i.e. for $-0.57 \leqslant \alpha \leqslant 2.34$
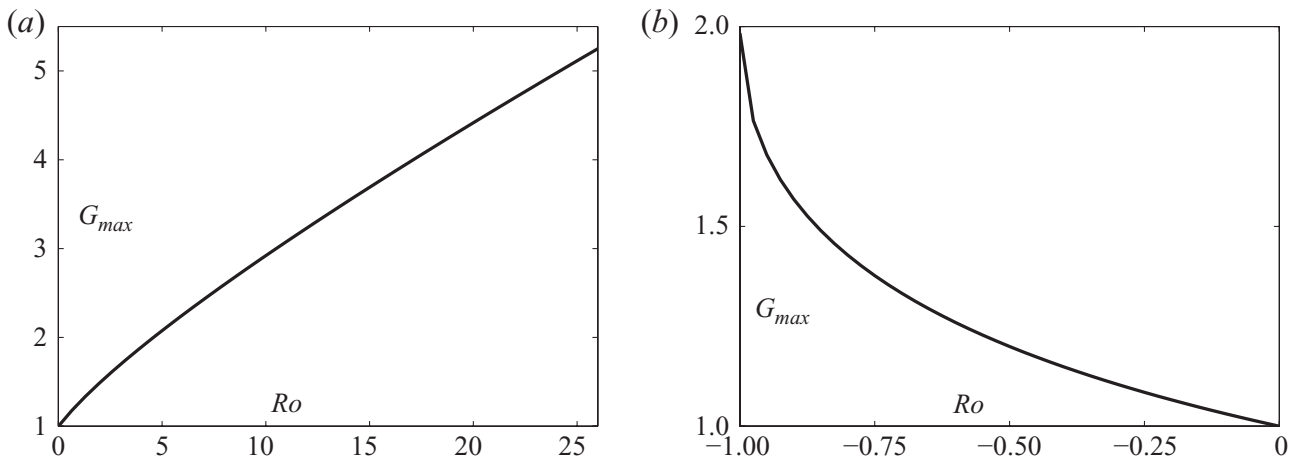

FIGURE 8. Upper bound $G_{\max }$ for the gain $G(t)=E(t) / E(0)$ for $(a)$ the cyclonic Lamb-Oseen vortex for the Lyapunov stable range of Rossby numbers $0<R o \lesssim 26$ with $G_{\max }=\max \{\mathscr{F}\}$ and $(b)$ for the anticyclonic Lamb-Oseen vortex in the Lyapunov and classically stable range $-1<R o<0$ with $G_{\max }=1 / \min \{\mathscr{F}\}$. For $R o=26$ we have $G_{\max } \approx 5.25$ in $(a)$ while for $R o \downarrow-1$ in $(b) G_{\max } \rightarrow 2$. The bounds are valid for any Reynolds number.

second condition in (1.5) is satisfied for all Rossby numbers in the classically stable range $-1<R o<0$. This is illustrated in figure $6(b)$ with the examples $R o=-0.9$ and $R o=-1 / 2$. A logarithmic scale is used along the vertical axis because of the large differences between the peak values of $(\mathrm{d} \mathscr{F} / \mathrm{d} r)^{2} /(\mathscr{F})^{2}$ as the Rossby number varies. So, contrary to the cyclonic case, (1.5) proves Lyapunov stability of the anticyclonic Lamb-Oseen vortex in the viscous dynamics for all Rossby numbers in the classically stable range.

We find that (1.4) also proves stability for the entire classically stable range: for all Rossby numbers in this range $(-1<R o<0)$ there is always an $\alpha \in[-1,3]$ for which $\mathscr{G} \leqslant 0$ for all $r$. Figure 7 shows two examples: in figure $7(a)$ the Rossby number is $R o=-0.99$, in figure 7(b) $R o=-1 / 2$. In both cases there is a negative and positive 
$\alpha$ (indicated by the thick lines) for which $\mathscr{G}=0$ at some finite $r$. For all $\alpha$ in between these two extremes, $\mathscr{G}<0$ for all finite $r$ and only asymptotically $\mathscr{G} \rightarrow 0$ as $r \rightarrow \infty$. For $R o=-0.99$ Lyapunov stability follows for $-0.57 \lesssim \alpha \lesssim 2.34$ (see figure $7 a$ ), for $R o=-1 / 2$ stability for $-0.74 \lesssim \alpha \lesssim 2.66$ (see figure $7 b$ ). The smallest range of $\alpha$ values with which Lyapunov stability follows is found in the limit $R o \downarrow-1$.

The bound $G_{\max }$ on the gain is shown in figure $8(b)$. Since $0<\mathscr{F} \leqslant 1$ in this case, according to $(2.21 a) G_{\max }=1 / \min \{\mathscr{F}\}$. In the entire range $-1<R o<0, G_{\max }$ never exceeds a value of 2 . Hence just as in the cyclonic case, no significant transient growth can be expected for the anticyclonic Lamb-Oseen vortex with Rossby numbers in the stable range, no matter how large the Reynolds number is.

\section{Summary and discussion}

In this paper we have first shown that if the inviscid classical criterion for symmetric stability is satisfied $(\mathscr{F}(r)>0$ or $\Phi(r)>0$ everywhere), the effective energy $\mathscr{E}$ defined in (2.11) or (2.12) is a Lyapunov functional. It establishes Lyapunov stability in the inviscid dynamics for arbitrary circular flows with respect to circularly symmetric perturbations. In the inviscid dynamics $\mathrm{d} \mathscr{E} / \mathrm{d} t=0$ and we showed that $G_{\max }$ in $(2.21 a)-(2.21 c)$ provides an upper bound for the amplification of perturbation energy which may occur due to transient non-modal growth in the inviscid dynamics. The development is somewhat simpler but otherwise analogous to the case of parallel shear flows in stratified fluids as discussed in Kloosterziel \& Carnevale (2007).

Next we have derived two novel criteria for Lyapunov stability of viscous circular flows with respect to symmetric perturbations, i.e. (1.4) and (1.5). In both criteria we first find the requirement for classical inviscid stability, i.e. $\Phi>0$ or $\mathscr{F}>0$ which guarantees that $\mathscr{E}$ is positive definite. The additional conditions guarantee that in the viscous dynamics $\mathrm{d} \mathscr{E} / \mathrm{d} t \leqslant 0$ at all times. This implies that the upper bound $G_{\max }$ for the gain in the inviscid dynamics remains valid for the viscous dynamics. If the second conditions in (1.4) and (1.5) are not satisfied in some overlapping regions, there is the possibility that the effective energy grows for some period of time, i.e. $\mathscr{E}(t)>\mathscr{E}_{0}$ and then $(2.21 a)-(2.21 c)$ may not be true.

The theory has been applied to a few examples. For other types of vortices or confined circular flows than discussed in this paper, one must apply both (1.4) and (1.5) to see if the flow is provable stable. If a flow is characterized by a variable parameter like the Rossby number in rotating systems or some parameter which determines the velocity distribution and is provable stable with both (1.4) and (1.5), the results must be compared to see which of the two criteria proves stability for the broadest range of the parameter(s). It is impossible to predict a priori.

For Couette flow we established in $\S 3$ with (1.4) Lyapunov stability for the entire classically stable range by setting $\alpha=3$. A normal-modes analysis by Synge (1938) and an energy method by Wood (1964) had already established this, but both studies were specifically aimed at Couette flow. In this study it quickly followed with the general condition (1.4) which can be applied to any flow for which $Q \neq 0$ everywhere.

In $\S 4.1$ we found it impossible to prove Lyapunov stability of the Lamb-Oseen vortex in a non-rotating system $(f=0)$, both in the inviscid and the viscous dynamics. For the inviscid case this is not surprising because for large $r$ the Lamb-Oseen vortex approaches potential flow, i.e. according to (4.1) for large $r$ we have approximately $V(r) \approx \omega_{0} L^{2} / r$ so that $Q \approx 0$. As noted by Miyazaki \& Hunt (2000), potential flow supports unbounded algebraic growth. This is easily seen by setting $v=0$ and $f=0$ in (2.3) so that $\partial v / \mathrm{d} t=0$ when $V \propto 1 / r$. Hence for potential flow $v(r, z, t)=v_{0}(r, z)$ 
remains unchanged. This can drive growth of the azimuthal vorticity $\omega_{\theta}$ in the linearized dynamics according to

$$
\frac{\partial \omega_{\theta}}{\partial t}=\frac{\partial}{\partial t}\left[\frac{\partial u}{\partial z}-\frac{\partial w}{\partial r}\right]=\frac{2 V}{r} \frac{\partial v_{0}}{\partial z}
$$

provided that $\partial v_{0} / \partial z$ does not vanish everywhere. Therefore the perturbation kinetic energy can grow without bounds if $\partial v_{0} / \partial z \neq 0$. Pradeep \& Hussain (2006) also noted that in the inviscid case the Lamb-Oseen vortex can experience amplification of perturbation energy that becomes unbounded as the 'optimal' perturbations are initiated at ever increasing distances from the vortex axis. Our thought experiment in $\S 2.2$, which showed how $G_{\max }=\max \{\mathscr{F}\}$ in $(2.21 b)$ might be approached, agrees with their observation that for initial perturbations concentrated at ever larger $r$, an ever increasing gain is expected. In the viscous case, Pradeep \& Hussain (2006) calculated a maximal gain of about $G_{\max }=10^{2}$ for $R e=2500$ which will continue to increase indefinitely with increasing Reynolds number. With our approach we could not establish a finite upper bound for the gain because for large $r$ both (1.4) and (1.5) are not satisfied so that $(2.21 a)-(2.21 c)$ may be false.

In $\S 4.2$ (1.5) proved stability of the cyclonic Lamb-Oseen vortex in the viscous dynamics for a finite range of positive Rossby numbers $0<R o \lesssim 26$ whereas there is classical stability for all $R o>0$. With (1.4) stability followed for a smaller range of Rossby numbers. For $0<R o \lesssim 26$ we found $1<G_{\max } \lesssim 5.25$. Our results imply that even in the case of rather weak rotation (large positive $R o$ but $R o<26$ or small 'rotation number' $1 / R o$ ) a numerical search for significant gain (several orders of magnitude) in perturbation energy would be futile no matter how large the Reynolds number is.

For $R o>26$ we do not expect a sudden transition to far larger transient growth than for $R o \approx 26$ because there is no destabilizing mechanism present like the doublediffusive mechanism for baroclinic flows mentioned in the introduction. Since ever increasing $R o$ can be interpreted as ever weaker rotation, the computations by Pradeep \& Hussain (2006) for the non-rotating case suggest that as Ro tends to infinity, for finite Reynolds numbers the maximum gain gradually approaches some finite value $G_{\max }(R e)$ and $G_{\max } \rightarrow \infty$ as $R e \rightarrow \infty$. For the anticyclonic Lamb-Oseen vortex we found in $\S 4.3$ that either condition (1.4) or (1.5) established stability in the viscous dynamics for the entire classically stable range $-1<R o<0$ with $1<G_{\max }<2$.

We must note that a study of the evolution of an integral quantity like the effective energy $\mathscr{E}$ cannot predict what kind of perturbations, characterized by spatial structure and relative amplitudes of the three perturbation velocity components, lead to maximal gain in the perturbation energy $E$. This can only be determined with a numerical search procedure as described by Pradeep \& Hussain (2006). For inviscid flows likely candidates are perturbations concentrated about the maximum or minimum of $\mathscr{F}$ but for viscous flows further analysis is required.

The modest upper bounds for the possible gain in the case of the Lamb-Oseen vortex in a rotating environment can be understood with simple model which captures the inviscid behaviour. The model equations are

$$
\frac{\partial u}{\partial t}=\left(\frac{2 V}{r}+f\right) v, \quad \frac{\partial v}{\partial t}=-\left(\frac{\mathrm{d} V}{\mathrm{~d} r}+\frac{V}{r}+f\right) u=-Q u
$$


which follow from (2.2) and (2.3) by ignoring pressure perturbations. If we solve (5.2) for some position $(r, z)$, the general solution is

$$
u(t)=u_{0} \cos (\sqrt{\Phi} t)+v_{0} \sqrt{\mathscr{F}} \sin (\sqrt{\Phi} t), \quad v(t)=v_{0} \cos (\sqrt{\Phi} t)-\frac{u_{0} \sin (\sqrt{\Phi} t)}{\sqrt{\mathscr{F}}} .
$$

If $V=0$ and $f \neq 0$ one has $\mathscr{F}=1$ and $\Phi=f^{2}$ and (5.3) describes simple inertial oscillations. The effective energy for this model is conserved, i.e. $\mathscr{E}(t)=$ $(1 / 2)\left(u^{2}(t)+\mathscr{F} v^{2}(t)\right)=(1 / 2)\left(u_{0}^{2}+\mathscr{F} v_{0}^{2}\right)=\mathscr{E}_{0}$ but the kinetic energy is not unless $\mathscr{F}=1$. If one chooses a location where $\mathscr{F}>1$ and takes $u_{0}=0$, then $E_{0}=(1 / 2) v_{0}^{2}$ but at time $t=2 /(\sqrt{\Phi} \pi)$ we have $E(t)=(1 / 2) \mathscr{F} v_{0}^{2}$ and the 'gain' is therefore $\mathscr{F}>1$. If $\mathscr{F}<1$ a gain of $1 / \mathscr{F}>1$ is found by taking $v_{0}=0$. This is essentially a mathematical formulation of our thought experiment at the end of $\S 2.2$ which made it plausible that the upper bounds $(2.21 a)$ and $(2.21 b)$ are fairly sharp in the inviscid dynamics.

A Taylor-series expansion yields

$$
u(t)=u_{0}+v_{0}\left(\frac{2 V}{r}+f\right) t-\frac{1}{2} u_{0} \Phi t^{2}+\ldots, \quad v(t)=v_{0}-u_{0} Q t-\frac{1}{2} v_{0} \Phi t^{2}+\ldots
$$

The higher order terms (proportional to $t^{3}, t^{4}$, etc.) vanish in the limit $Q \rightarrow 0$ in which case also $\Phi \rightarrow 0$. The algebraic growth for potential flow $V \propto 1 / r$ when $f=0$, which means $Q=0$, is recovered provided that $v_{0} \neq 0$. When $f \neq 0$, the $u$ and $v$ field are coupled through the Coriolis force and generally this leads to oscillatory behaviour.

For large $r$ in the Lamb-Oseen vortex $Q \approx 0$ while $\mathscr{F} \gg 1$ and large amplification is possible but the growth is very slow because $\Phi \approx 0$. For the Lamb-Oseen vortex in a rotating environment we have $\Phi>0$ for all $R o>-1$. For the cyclone $\Phi \geqslant f^{2}$ whereas for the anticyclone $\Phi \geqslant f^{2}(1-|R o|)^{2}$. In the context of the simple model this means that for the cyclone the time scale $T_{\max }$ for the transient amplification is faster than $1 / f$, i.e. $T_{\max }<1 / f$ and for the anticyclone $T_{\max }<1 /(f(1-|R o|))$. A model with Rayleigh damping added to (5.2) has been discussed by Pradeep \& Hussain (2006, equation (4.2)). This is a reasonable model for the viscous dynamics. It is clear that with damping the maximum gain will be smaller and occur at an earlier time than in the undamped dynamics. For our simple model this means that if damping is added, the time scale $T_{\max }$ remains smaller than $1 / f$ for the cyclonic Lamb-Oseen vortex and smaller than $1 /(f(1-|R o|))$ for the anticyclone.

As is quite common in viscous stability studies, we assumed that the basic flow $V(r)$ is stationary in the viscous dynamics. This is true for Couette flow, solid body rotation and potential flow but not, for example, for the Lamb-Oseen vortex. This was not mentioned by Pradeep \& Hussain (2006) and various others preceding them with studies of viscous transient growth. If we imagine the presence of a circularly symmetric force field acting in the azimuthal direction, i.e. a force $F_{\theta}(r)=-\rho \nu \Delta_{1} V(r)$ then any flow would be stationary. For such a 'forced-dissipative' system, our results remain valid. But for freely evolving viscous flows like the unforced Lamb-Oseen vortex, the time-dependence may be important. The time scale of evolution of the Lamb-Oseen vortex is $T_{v}=L^{2} / v$, where $L$ is roughly the initial 'size' of the vortex (the distance from the axis where $V=V_{\max }$; see figure $3 a$ ). If we take as the relevant time scale for the growth of perturbations the transient growth time scale $T_{\max }$ then the assumption of stationarity is valid if $T_{v} / T_{\max } \gg 1$. Using the time scale from our model for $T_{\max }$, we expect transient growth to be much faster than the viscous 
evolution of the cyclone when $R e \gg R o$ while for the anticyclone the model suggest that it requires $R e \gg|R o| /(1-|R o|)$. For any Rossby number $R o>-1$ this can be satisfied if $R e$ is sufficiently large. The favourable comparison found by Pradeep $\&$ Hussain (2006, figure 14) between the numerically determined optimal gains $G_{\max }$ and the time scale $T_{\max }$ and the $G_{\max }$ and $T_{\max }$ found with the simple model with Rayleigh damping, suggests that at least for large-Reynolds-number flows our results are valid.

A discussion of separation of time scales is unnecessary if general criteria can be formulated that guarantee Lyapunov stability in the viscous dynamics for arbitrary time-dependent barotropic flows $V=V(r, t)$ but this has not yet been accomplished. Another obvious extension of this work is to consider what are the stability criteria for viscous symmetric stability for stationary baroclinic flows $V(r, z)$ in stratified and rotating fluids and non-stationary flows $V(r, z, t)$. Also of considerable interest in geophysical fluid dynamics is symmetric stability/instability of zonal flows on the equatorial $\beta$ plane. This was already considered by Kloosterziel \& Carnevale (2007) but only for a particularly simple flow. Whether sufficient conditions for viscous Lyapunov stability can be found for the above mentioned flows is currently an open question.

This material is based upon work supported by the National Science Foundation Grant OCE 07-26866. The author would like to express his gratitude to G. F. Carnevale for carefully reading the manuscript.

\section{Appendix. Normal-modes analysis}

If normal-modes solutions of the linear perturbation equations exist, we can derive the inviscid classical criterion for stability $(\Phi(r)>0$ everywhere) as follows. Eliminating the pressure perturbation between (2.2) and (2.4) and setting $v=0$, we get the equation for the meridional vorticity component $\omega_{\theta}=\partial_{z} u-\partial_{r} w$ :

$$
\frac{\partial}{\partial t}\left[\frac{\partial u}{\partial z}-\frac{\partial w}{\partial r}\right]-\left(\frac{2 V}{r}+f\right) \frac{\partial v}{\partial z}=0 .
$$

Continuity (2.5) will be satisfied if we introduce a streamfunction for the motions in the meridional $r z$ plane: $u=\partial \psi / \partial z, w=-(1 / r) \partial r \psi / \partial r$. Differentiating (A 1) with respect to time and substituting $\partial v / \partial t$ from (2.3) with $v=0$, we find

$$
\frac{\partial^{2}}{\partial t^{2}}\left[\frac{\partial}{\partial r} \frac{1}{r} \frac{\partial r \psi}{\partial r}+\frac{\partial^{2} \psi}{\partial z^{2}}\right]+\Phi \frac{\partial^{2} \psi}{\partial z^{2}}=0
$$

This is a simple case of what meteorologists sometimes call the Eliassen-Sawyer equation (Sawyer 1949; Eliassen 1951).

The normal-modes assumption is that we presume that solutions $\psi=\mathrm{e}^{s t} \Psi(r, z)$ exist. For convenience we assume that the flow in the meridional plane is bounded by rigid boundaries (e.g. at $z=0$ and $z=H$ and at some radius $r=R$ ). The no-flux condition is satisfied if we set $\Psi=0$ at such boundaries. $\Psi$ may be complex and if so $\Psi^{\star}$ ( $\star$ indicates complex conjugate) must also vanish at the boundaries. We replace $\partial^{2} / \partial t^{2}$ by $s^{2}$ in (A 2), $\psi$ by $\Psi$ and then multiply by $\Psi^{\star}$. Integrating over the domain we find, using the boundary condition for $\Psi$ and $\Psi^{\star}$, that

$$
s^{2}=-\int_{\mathscr{V}} \Phi\left|\frac{\partial \Psi}{\partial z}\right|^{2} \mathrm{~d} \mathscr{V} / \int_{\mathscr{V}}\left(\left|\frac{1}{r} \frac{\partial \Psi r}{\partial r}\right|^{2}+\left|\frac{\partial \Psi}{\partial z}\right|^{2}\right) \mathrm{d} \mathscr{V}
$$


If $\Phi(r)>0$ everywhere, $s^{2}<0$ for all normal-modes perturbations and there will be no growth. Hence stability is established in the inviscid dynamics with the somewhat tenuous assumption that normal modes exist. The modes oscillate in that case without decay or growth. In a rotating system at rest $(V=0)$ we have $\Phi=f^{2}$ so that for the oscillation frequency $\omega$ (with $s= \pm \mathrm{i} \omega$ ) we find $0<|\omega|<|f|$. This corresponds to gyroscopic (internal) waves in a rotating fluid.

If we let $v \neq 0$ things become complicated. Instead of (A 2) we find

$$
D_{t}^{v}\left(\frac{D_{t}^{v} \Delta_{1} \psi}{2 V / r+f}\right)+\left(\frac{\mathrm{d} V}{\mathrm{~d} r}+\frac{V}{r}+f\right) \frac{\partial^{2} \psi}{\partial z^{2}}=0, \quad \text { where } \quad D_{t}^{v}=\partial_{t}-v \Delta_{1}
$$

with $\Delta_{1}$ defined in (2.7). If we set $v=0$ and if $2 V / r+f \neq 0$, this reduces to (A 2). For the $u$ field the same equation can also be derived, for the $w$ field we have failed to find a single equation when $v \neq 0$ and for the $v$ field the equation is

$$
D_{t}^{v} \Delta_{1}\left(\frac{D_{t}^{v} v}{\mathrm{~d} V / \mathrm{d} r+V / r+f}\right)+\left(\frac{2 V}{r}+f\right) \frac{\partial^{2} v}{\partial z^{2}}=0 .
$$

To proceed with a normal-modes analysis, a number of boundary conditions need to be supplied but we have found it impossible to prove with either (A 4) or (A 5) that generally there will be normal-modes stability if $v \neq 0$ and $\Phi>0$ everywhere. Normal-modes stability would follow only in certain special cases. For example, (A 5) with $f=0$ could prove normal-modes stability of Couette flow, at least for the $v$ field, if $\Phi>0$ everywhere. This would be an alternative to Synge's analysis who studied the normal-modes stability of Couette flow through two coupled equations that are first order in time (Synge 1938). For all flows for which either $V / r \neq$ constant in (A 4) or $\omega=\mathrm{d} V / \mathrm{d} r+V / r \neq$ constant in (A 5), mathematical difficulties arise which make it impossible to derive general conditions for normal-modes stability in the viscous dynamics.

\section{REFERENCES}

ARNOL'D, V. I. 1966 On an a priori estimate in the theory of hydrodynamical stability. Izv. Vyssh. Uchebn. Zaved. Mat. 54 (5), 3-5. Translation in 1969 Am. Math. Soc. Transl. Ser. 2 79, 267-269.

BAYLY, B. J. 1988 Three-dimensional centrifugal-type instabilities in inviscid two-dimensional flows. Phys. Fluids 31, 56-64.

Butler, K. M. \& Farrell, B. F. 1992 Three-dimensional optimal perturbations in viscous shear flow. Phys. Fluids A 4 (8), 1637-1650.

Carnevale, G. F., Briscolini, M., Kloosterziel, R. C. \& Vallis, G. K. 1997 Three-dimensionally perturbed vortex tubes in a rotating flow. J. Fluid Mech. 341, 127-163.

Chandrasekhar, S. 1961 Hydrodynamic and Hydromagnetic Stability. Oxford University Press.

Charney, J. G. 1973 Lecture notes on planetary fluid dynamics. In Dynamic Meteorology (ed. P. Morel), pp. 97-352. Reidel.

Cho, H.-R., Shepherd, T. G. \& Vladimirov, V. A. 1993 Application of the direct Liapunov method to the problem of symmetric stability in the atmosphere. J. Atmos. Sci. 50, 822-836.

Drazin, P. \& ReID, W. 1981 Hydrodynamic Stability. Cambridge University Press.

Eliassen, A. 1951 Slow thermally or frictionally controlled meridional circulation in a circular vortex. Astrophys. Norvegia 5, 19-60.

Eliassen, A. \& Kleinschmidt, E. 1957 Dynamic meteorology. In Handbuch der Physik (ed. J. Bartels), vol. 48, pp. 1-154. Springer.

Farrell, B. F. 1988 Optimal excitation of perturbations in viscous shear flow. Phys. Fluids 31 (8), 2093-2102.

FJøRTOFт, R. 1950 Application of integral theorems in deriving criteria of stability for laminar flows and for the baroclinic vortex. Geofys. Publ. 17 (6), 5-52. 
HøIland, E. 1962 Discussion of a hyperbolic equation relating to inertia and gravitational fluid oscillations. Geofys. Publ. 24, 211-227.

Kloosterziel, R. C. \& Carnevale, G. F. 2007 Generalized energetics for inertially stable parallel shear flows. J. Fluid Mech. 585, 117-126.

Kloosterziel, R. C., Carnevale, G. F. \& Orlandi, P. 2007 Inertial instability in rotating and stratified fluids: barotropic vortices. J. Fluid Mech. 583, 379-412.

Kloosterziel, R. C. \& van Heisst, G. J. F. 1991 An experimental study of unstable barotropic vortices in a rotating fluid. J. Fluid Mech. 223, 1-24.

McIntyre, M. E. 1970 Diffusive destabilization of the baroclinic circular vortex. Geophys. Fluid Dyn. 1, 19-58.

van Mieghem, J. M. 1951 Hydrodynamic instability. In Compendium of Meteorology (ed. T. F. Malone), pp. 434-453. American Meteorological Society.

MiYAZAKI, T. \& Hunt, J. C. R. 2000 Linear and nonlinear interactions between a columnar vortex and external turbulence. J. Fluid Mech. 402, 349-378.

Mu, M., Shepherd, T. G. \& Swanson, K. 1996 On nonlinear symmetric stability and the nonlinear saturation of symmetric instability. J. Atmos. Sci 53, 2918-2923.

Ooyama, K. 1966 On the stability of the baroclinic circular vortex: a sufficient condition for instability. J. Atmos. Sci. 23, 43-53.

Pradeep, D. S. \& Hussain, F. 2006 Transient growth of perturbations in a vortex column. J. Fluid. Mech. 550, 251-288.

RaYleigh, J. W. S. 1916 On the dynamics of revolving fluids. Proc. R. Soc. Lond. A 93, 148-154.

SAwYer, S. J. 1947 Notes on the theory of tropical cyclones. Quart. J. R. Met. Soc. 73, 101-126.

SAWYER, S. J. 1949 The significance of dynamic instability in atmospheric motions. Quart. J. R. Met. Soc. 75, 364-374.

Schmidt, P. J. 2007 Nonmodal stability theory. Ann. Rev. Fluid Mech. 39, 129-162.

SIPP, D. \& JACQUIN, L. 2000 Three-dimensional centrifugal-type instabilities in inviscid twodimensional flows in rotating systems. Phys. Fluids A 12, 1740-1748.

SolberG, H. 1936 Le mouvement d'inertie de l'atmosphere stable et son role dans la theorie des cyclones. Sixth Assembly, Edinburgh. Union Geodesique et Geophysique Internationale, 66-82.

Synge, J. L. 1938 On the stability of a viscous liquid between rotating coaxial cylinders. Proc. Roy. Soc. A 167, 250-256.

Trefethen, L. N., Trefethen, A. E., Reddy, S. C. \& Driscoll, T. A. 1993 Hydrodynamic stability without eigenvalues. Science 261, 578-584.

Wood, W. W. 1964 Stability of viscous flow between rotating cylinders. ZAMP 15 (3), 313-314.

YANAI, M. \& ToKiAKA, T. 1969 Axially symmetric meridional motions in the baroclinic circular vortex: a numerical experiment. J. Meteorol. Soc. Japan 47 (3), 183-197. 